\title{
Stresses in granular materials
}

\author{
David Muir Wood • Danuta Leśniewska
}

Received: 31 December 2009 / Published online: 15 December 2010

(C) The Author(s) 2010. This article is published with open access at Springerlink.com

\begin{abstract}
When circularly polarised light is passed through a granular material under boundary stresses patterns- 'light stripes' - are seen in the resulting images which have been traditionally associated with the directions of major principal stresses in the equivalent continuum. In this paper the passage of polarised light through a single spherical particle under stress is studied experimentally and analytically. The effect of placing the particle within a layer of particles, a layer of thickness $2-3$ particles, and within a mass of particles is investigated experimentally. The appearance of light stripes is a visual reinforcement of effects seen at the particle level provided the level of stresses in individual particles is low. The implications for quantitative photoelastic interpretation of granular media are discussed.
\end{abstract}

Keywords Glass particles · Photoelasticity ·

Stress distribution $\cdot$ Particulate material

D. Muir Wood

Division of Civil Engineering, University of Dundee,

Fulton Building, Dundee DD1 4HN, UK

e-mail: d.muirwood@dundee.ac.uk

D. Muir Wood $(\varangle)$

Department of Civil Engineering, University of Bristol,

Queen's Building, University Walk, Bristol BS8 1TR, UK

D. Leśniewska

Polish Academy of Sciences, Institute of Hydro-Engineering,

Kościerska 7, 80-328 Gdańsk, Poland

e-mail: d.lesniewska@ibwpan.gda.pl

D. Leśniewska

Koszalin University of Technology, Śniadeckich 2, 75-453 Koszalin, Poland

\section{Introduction}

Photoelasticity is one of a number of experimental methods which use light as an information carrier. It belongs to so called 'full field' optical methods [24], in which spatially extended light beams propagate in free space, illuminating and creating images of entire objects. Polarised light is used for photoelastic methods.

When birefringent materials are viewed using polarised light, patterns can be observed which are linked to the stress state in the material. Photoelastic studies of models of structural elements are used to reveal the internal stress distributions. A mass of particles of birefringent material subjected to boundary stresses also shows structured information which is related to the applied stresses but the detailed interpretation of this information is less clear, in part because the continuum estimate of macroscopic stress is quite different from the actual stresses experienced within the individual particles.

'Experts in soil mechanics use Coulomb's law by assuming that the shear stress $\tau$ is related to the normal stress $\sigma_{n}$ by $\tau=\mu_{s} \sigma_{n}$. In doing so they implicitly admit that a stress tensor can be defined in a granular material (which is far from evident) [...] The assumption turns out to be justified in most situations.' [7]. The use of Mohr-Coulomb descriptions of failure of soils is confidently applied at the macroscopic scale where the sample or geotechnical prototype is considerably larger than an individual particle and we can reasonably treat the soil as a continuum.

However, photoelastic studies on granular materials have, perforce, in many cases been performed using small numbers of particles. For Duran [7], a 'small number' of particles means fewer than 8,000 but analysis of strain features in sands and other granular materials $[13,17]$ suggests that the frame of observation should have all dimensions of at least $\sim 300$ particles so that a two-dimensional section would require 
some $300 \times 300 \approx 10^{5}$ particles and a three-dimensional assembly would require some $300 \times 300 \times 300 \approx 3 \times 10^{7}$ particles in order to smear out the patterns of localised deformation that are observed. It seems likely that the interpretation of test results must account for the size of the granular assembly and its degree of spatial order and disorder.

Tests described by Leśniewska and Muir Wood [15] were performed on 'large' particle assemblies (containing typically of the order of $10^{6}$ glass particles in a sample with section roughly $180 \times 230 \approx 4 \times 10^{4}$ particles with thickness around 20 particles - though this is of course still much smaller than the number of particles in a full-scale geotechnical structure). Linking photoelastic observations with fields of strains deduced from particle movements revealed some features (especially at micro-scale), which have not been observed in previous photoelastic studies of granular materials. That paper provided some initial results and thoughts concerning the possibilities of parallel viewing of an assembly of glass particles under polarised light and under unpolarised light with the conclusion that it was certainly possible to extract information about the displacement and strain fields and also possible to extract some information concerning the stress conditions within the granular sample. However, the present paper explores more fully the theoretical possibilities and implications of passing polarised light through an assembly of glass particles subjected to some external stress field.

We will show how the features seen in a large granular assembly can be related to the features seen in a loaded single particle having dimensions in the range of typical sand grains $(\sim 1 \mathrm{~mm})$. From the photoelastic effects in such tiny objects we can deduce possible implications of the micro scale effect on the whole sample response. We will also point out the specific problems related to the interpretation of photoelastic observations obtained from large assemblies of three dimensional grains - the methods of analysis commonly used in continuum photoelasticity cannot be automatically employed for such assemblies.

\section{Interpretation of photoelastic images}

Figure 1 belongs to the class of transmission images which start with a uniform light source of known intensity and colour. Such images show the intensity of light (or other radiation) that has come through the sample [19]. The intensity of transmission of the light at each point is a measure of the photoelastic effect in the specimen along that path. The use and interpretation of photoelastic images of granular materials have been discussed in several classic papers: Dantu [4], Wakabayashi [26], Drescher and De Josselin de Jong [6], Drescher [5], Dyer [8], Allersma [3], Liu et al. [16]. However, while each of these authors notes the 'light stripes' that are

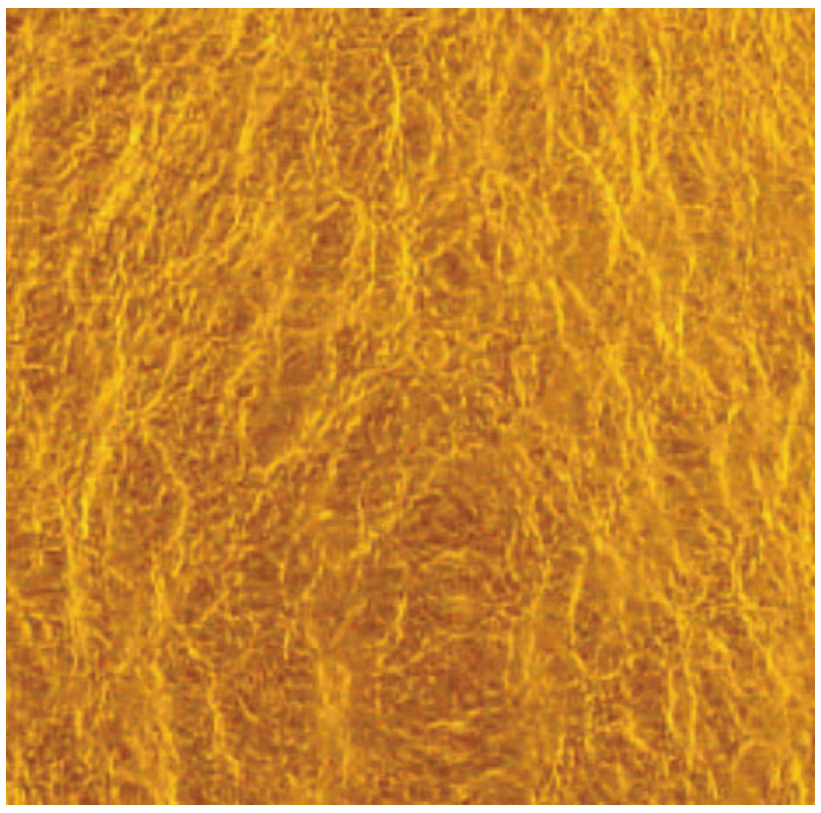

Fig. 1 Close-up of 'light stripes' seen in assembly of glass particles under polarised light. (For experimental configuration see Fig. 19.)

seen when loaded masses of appropriate granular materials are viewed with polarised light (e.g. Fig. 1), the physical interpretation of these features has largely been qualitative and somewhat uncertain.

The mathematical description of the behaviour of light in a double-refractive material presented in this paper is based on a plane stress model of photoelastic material, starting from the assumption that the material is mechanically and optically homogeneous and the stress distribution is plane and homogeneous over the sample thickness. In soil mechanics a model made of three dimensional grains is regarded as deforming in plane strain when it is placed in a rectangular container preventing any significant macroscopic deformation in the direction perpendicular to the plane of loading. Such model tests have been used to validate solutions of many standard soil mechanics problems. The plane strain constraint is an overall kinematic constraint for the assembly but individual particles will have no detailed awareness of the nature of those more or less distant boundaries and will feel themselves neither under plane stress nor under plane strain. Model tests $[3-5,8,26]$ have been made on samples built of a very large number of grains, such that the grain diameter is much smaller than the typical dimensions of the problem. These authors were aware that the assumptions of the photoelasticity theory, such as homogeneity of the sample and stress homogeneity over the sample thickness, were not fulfilled at the grain scale, but they assumed that they would nevertheless be fulfilled macroscopically: '...it is presumed that the optically determined stress components describe a two-dimensional stress state in the plane of the sample, which is in agreement with the aver- 
aged stress.' [3] Allersma's approach is equivalent to treating the macroscopically plane granular sample as a plate made of imperfect but uniform photoelastic material (with voids), but the imperfection is neglected in the applied optical measurements which have been developed for plane continuous material. While this may be satisfactory as a first step in studying granular materials by photoelasticity, more detailed consideration is required to take account of the double photoelasticity of the particles-at the macro scale (imperfect plate) and at the micro scale (each grain exhibits its individual photoelastic response). We can easily imagine that both phenomena can be present with different intensities, depending on the particular test conditions. The 'light stripes' described in classical papers on photoelastic granular materials definitely belong to phenomena at the grain scale.

The interpretation of light stripes or photoelastic force chains as principal stress trajectories comes from the simple reasoning, recalled by Allersma: 'Since the chains transmit the largest forces it can be reasonably supposed that the light stripes represent the principal stress trajectories.' Validation of this statement has been performed using photoelastic measurements based on the assumption that concepts taken from continuum photoelasticity would also be valid for granular materials. Not all available experimental data support such an interpretation. There still exist open questions regarding exact interpretation of previously investigated light stripes or force chains. The range of very small deformations was not accessible to previous researchers for technical reasons and it was not possible to extract information from photoelastic images before clear light stripes formed at large deformations.

Recently the use of photoelastic observations of granular materials (with small numbers of particles) has been taken up vigorously by Behringer and his colleagues (e.g. $[9,10,23])$ reporting studies of single layers of photoelastic discs made of polymer material under various classes of boundary loading. However, Fig. 2 comes from the pioneering work of Drescher and De Josselin de Jong [6]. Such photographs show very clearly that the boundary stresses are carried through chains of highly loaded particles, while some particles (which remain light in Fig. 2) carry little or no stress. Figure 3 uses lines of thickness proportional to the contact force between adjacent particles to join the centres of those particles. The strong force chains in this two-dimensional assembly that is being subjected to shear by rotation of the two visible inclined boundaries OA and OD are quite apparent. While these force chains cannot be described as straight or parallel it seems that there is a general dominant direction which is more or less aligned with the major principal stress direction deduced from a continuum interpretation of the discrete forces between the particles and the rigid boundaries. An exactly similar conclusion is regularly drawn from the results of discrete element analysis (DEM) (e.g. Fig. 4).

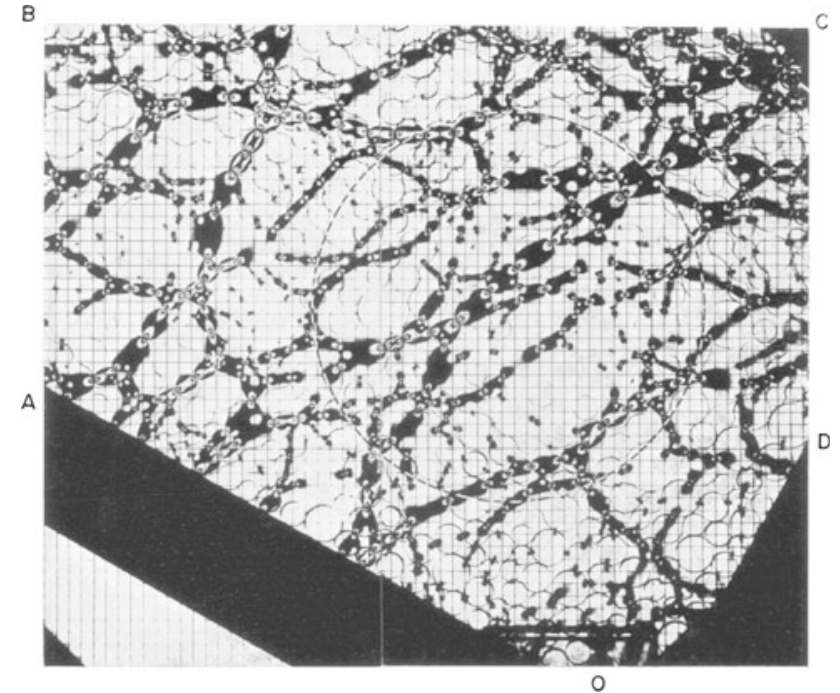

Fig. 2 Two-dimensional assembly of photoelastic discs under polarised light [6].

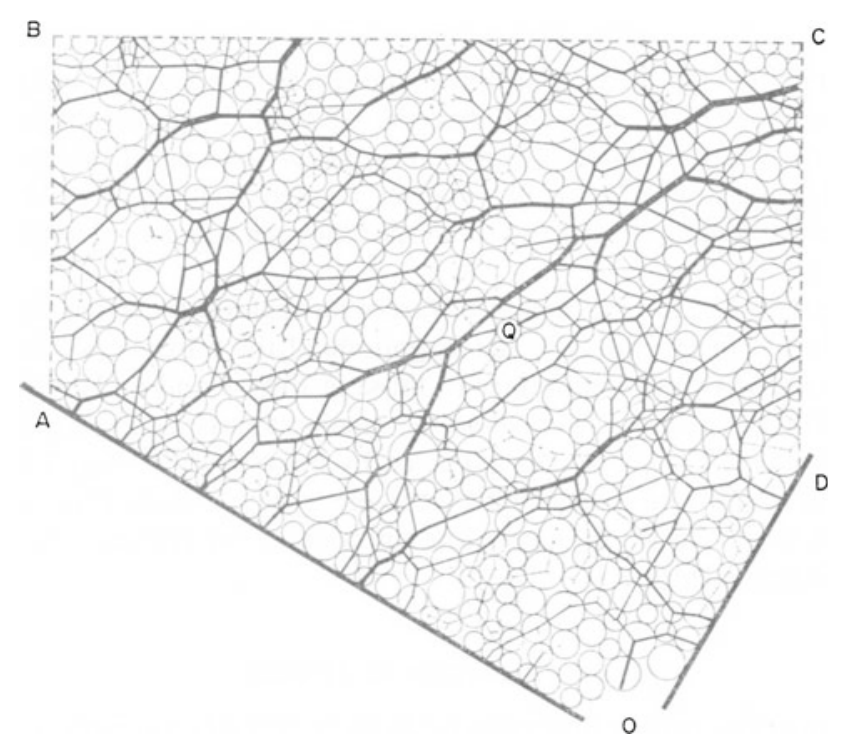

Fig. 3 Network of contact forces extracted from photoelastic image (Fig. 2) [6]

These strong force chains readily buckle and re-form when the direction of major principal stress is rotated. Many of the force chains observed seem to form closed loops, producing a characteristic cellular structure, visible in Figs. 2, 3 and 4, but also apparent at higher magnification in Fig. 1.

Tordesillas et al. [22] use photoelastic observation of assemblies of around 3,000 discs ('small' granular assemblies, according to the classification of Duran [7]) to extract information that can be used to inform the development of constitutive models based on particle level observations. 'To elucidate the mechanism for dissipation, we probe the behaviour of the force chain network where energy is primarily stored. Initially aligned with the major principal (most 


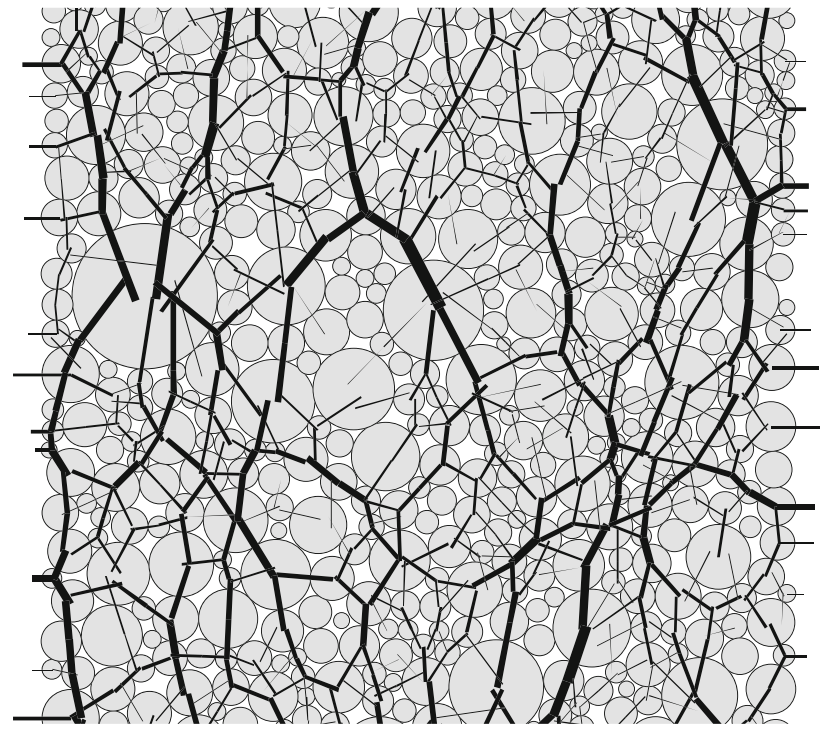

Fig. 4 Network of contact forces in discrete element analysis of twodimensional assembly of discs (Maeda, personal communication)

compressive) stress axis, force chains accumulate stored potential energy, concurrent with the steady growth of the normal contact forces during the jamming stages or rise in stress ratio.' [22] However, they suggest that the force chains linking the more heavily loaded particles are only initially aligned with the major principal stresses.

Both numerical simulations and experiments (for example, Geng et al. [10]) show that, as one might expect, repetition of experiments under identical macroscopic conditions-but different particle arrangements-typically leads to substantially different force chain patterns each time, especially when the granular sample consists of a limited number of grains-which is to say that the ratio of sample size to particle size is low. Geng et al. note that 'this large variability under repetition suggests that a statistical approach might be the most appropriate one. This approach might take the form of averaging a single realisation over large regions of space. ... [This] is implicit in typical macroscopic models of stress propagation for granular materials.' However, a threedimensional assembly of grains is not composed of a series of two-dimensional assemblies of grains.

This paper explores some of the related questions through the observation of single particles, single layers and multiple layers of spherical glass particles under polarised and nonpolarised light.

\section{Photoelasticity: circularly polarised light}

The refractive index of a birefringent material is modified slightly by the application of stress. The changes in refractive index are sufficiently small for it to be reasonable to assume

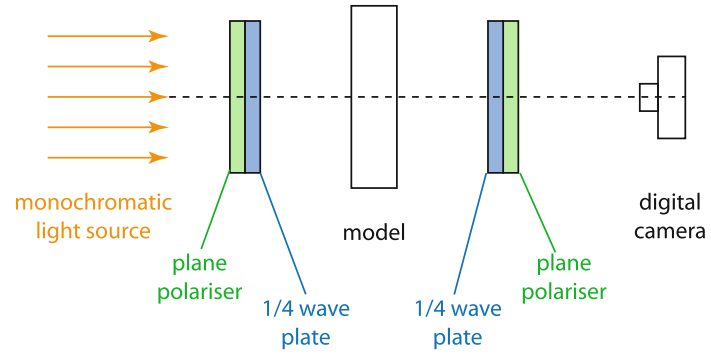

Fig. 5 Arrangement of circular polariscope

that a light ray passing through the material remains straight. The effects of such birefringence are best observed using circularly polarised light in a polariscope: a typical arrangement is shown in Fig. 5. A summary of relevant elements of analytical photoelasticity is contained in the Appendix: a more detailed treatment can be found in Ramesh [18], Aben [1], for example. Exactly the same polariser setup was used in all the two-dimensional and three-dimensional models presented here. The polarisers were of diameter $300 \mathrm{~mm}$ and were manufactured in the Institute of Fundamental Technical Research in Warsaw; they and the monochromatic sodium light sources have been used for scientific measurements performed over the past 40 years. The camera used for all the images, with polarised and unpolarised light, was a Sony Cybershot with resolution 2,560 x 1,920 pixels.

For light passing through a material under plane stress, the intensity $I_{q}$ of the emergent light that is perceived is:

$I_{q}=I_{a} \sin ^{2} \Delta / 2$

where $I_{a}$ accounts for the intensity of the incident light and $\Delta$ is a phase difference which is proportional to the local difference of principal stresses in the plane orthogonal to the light ray (12) (Appendix). An example of the relationship between light intensity and magnitude of the stress difference (12) (Appendix) at one point of a loaded piece of glass is shown in Fig. 6 for two different values of $C$ and for $h=8 \mathrm{~cm}$. The two values of $C$ represent the lower and upper limits established for common glass, according to Aben and Guillemet [2]. The higher the value of $C$ the more frequent the zero points for the light intensity function, resulting in alternating dark and bright fringes within the material as the load is increased. Correspondingly, the higher the value of $C$, the less monotonic the relationship between load and intensity of transmitted light.

The dark fringes in a piece of photoelastic material are easy to distinguish with the naked eye in contrast to the monochromatic ones which form a bright background. An example is shown in Fig. 7 for a plane glass disc of $20 \mathrm{~mm}$ diameter, $1.2 \mathrm{~mm}$ thick, under diametrically opposing loads. As the magnitude of the load is increased the disc first develops two bright regions immediately adjacent to the points of 


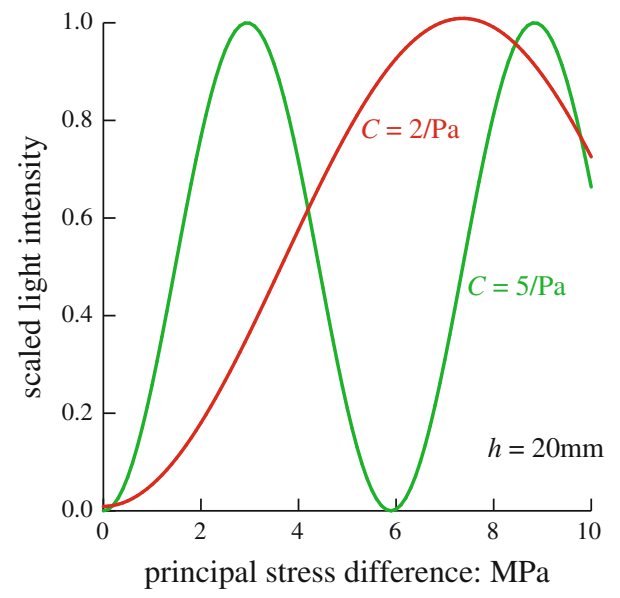

Fig. 6 Light intensity as a function of principal stress difference

application of the loads (Fig. 7 images 1 and 2); these then spread across the disc eventually filling its whole volume with monochromatic fringes (Fig. 7 images 3-7); then a first black fringe develops at the points of loading, corresponding roughly to $15 \%$ of the disc strength (Fig. 7 images 8-15) and extends across the disc forming a figure of eight when the two opposite black fringes merge, and then an ellipse as the load rises further. If the load is increased further still, additional monochromatic and dark fringes appear. Figure 8 shows the load-displacement curve obtained in one of four compression tests on glass discs identical to that shown in Fig. 7. The estimated load levels at which the successive dark fringes started to develop in Fig. 7 are also marked (points i-iv). The disc shown in Fig. 7 was broken when the fourth range of dark fringes was beginning to develop, after 50 load increments. The average disc strength was $38.27 \mathrm{MPa}$ (breaking force/diametral cross-section). The average stress increment $(0.8 \mathrm{MPa})$ could then be calculated by dividing the ultimate load by the number of load steps for each disc - thus neglecting the initial non-linearity of the load-displacement curve.

It can be seen from Fig. 7 that there exists a period in the loading history (images $8-15$ ), when an elongated bright fringe appears in the middle of the disc - its longer axis of symmetry obviously coincides with the direction of diametral loading. This direction could be properly deduced at this stage of the test by measuring the locations of maximum image intensity. However, at later stages of loading (images 16-50) detection of the maximum image intensity would be of no use, because darker regions appear along the loading axis as a result of natural fringe pattern evolution with increasing load. For a single disc (grain) or for small two dimensional assemblies of discs, symmetry of the fringe pattern could be used instead of light intensity to deduce the loading direction. The problem becomes much more complex when large, three-dimensional granular assemblies are considered and no individual fringe patterns can be traced. The validity of a method of finding principal stress directions

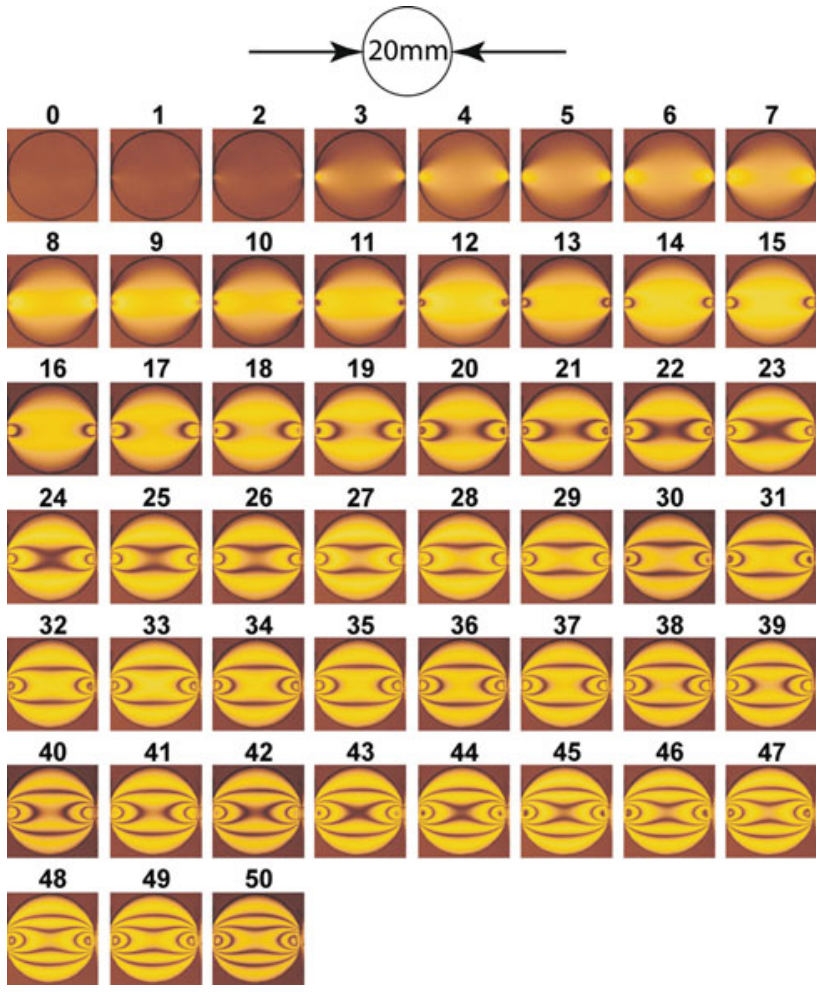

Fig. 7 Plane glass disc of $20 \mathrm{~mm}$ diameter, $1.2 \mathrm{~mm}$ thick, under diametrically opposing loads, viewed with circularly polarised light

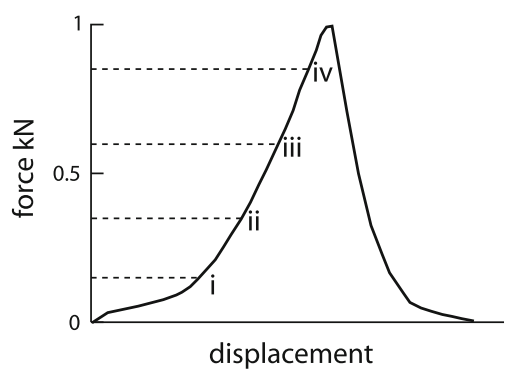

Fig. 8 Compression test on single plane glass disc of $20 \mathrm{~mm}$ diameter, $1.2 \mathrm{~mm}$ thick such as that shown in Fig. 7

based on the maximum image intensity must depend on the level of applied load, as Fig. 7 clearly shows.

Patterns of fringes, similar to those obtained in a single glass disc (Fig. 7) can be seen at the particle contacts in Fig. 2 (bright background 16) (Appendix). Some particles in this figure clearly show dark internal stripes because of the high stress level generated by the contact forces within individual particles. Whether we can reach contact forces in a granular assembly which take us beyond the monotonic link between intensity and load-beyond the first half fringe range - depends on the material of which the particles are made: the urethane rubber used by Utter and Behringer [23] is much more optically sensitive than the glass of Fig. 7 and produces fringes at much lower stress. 
(a)

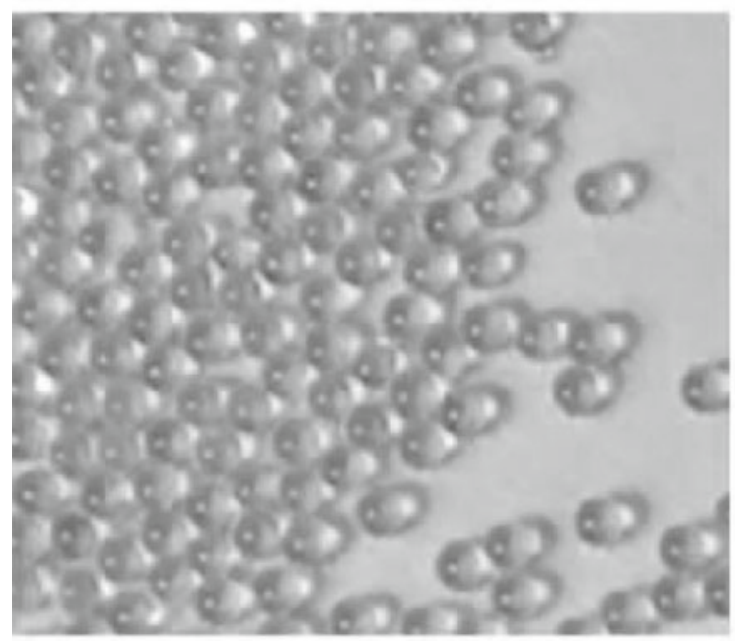

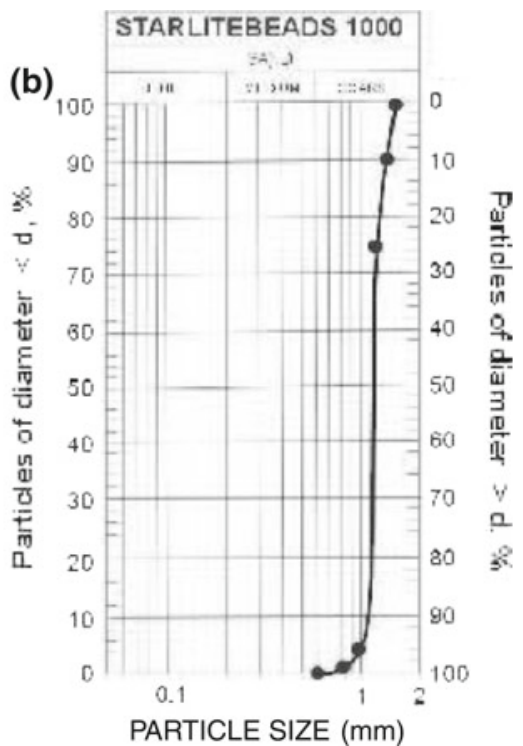

Fig. 9 Starlite beads

\subsection{Three dimensional states of stress}

Plane stress provides a simple case for which the effects of the internal material stresses on the transmission of polarised light can be readily calculated. However, in general we will be concerned with objects which are subjected to fully three-dimensional states of stress. According to Aben and Guillemet [2,p. 86] 'The simplest way to investigate stresses in a three-dimensional transparent specimen is to put it into an immersion bath and pass polarised light through it. Transformation of the state of polarisation which can be measured on many rays, contains information about the stresses in the model. This method is named integrated photoelasticity.'

The direction of passage of the light ray (the $z$-direction, for example) provides a reference axis which will in general not coincide with any of the principal axes of the three dimensional stress state. However, for the plane orthogonal to this ray, the $x y$ plane, there will be stresses $\sigma_{x x}, \sigma_{y y}, \tau_{x y}$ from which we can calculate 'secondary' principal stresses, $\sigma_{1}^{*}$ and $\sigma_{2}^{*},[18]$ :

$\begin{aligned} & \sigma_{1}^{*} \\ & \sigma_{2}^{*}\end{aligned}=\left(\sigma_{x x}+\sigma_{y y}\right) / 2 \pm \sqrt{\left[\left(\left(\sigma_{x x}-\sigma_{y y}\right) / 2\right)^{2}+\tau_{x y}^{2}\right]}$

with the principal direction of these 'secondary' principal stresses calculated at angle $\theta$ to the horizontal in the usual way:

$\tan \theta=2 \tau_{x y} /\left(\sigma_{x x}-\sigma_{y y}\right)$

The retardation $\delta$ can then be calculated from (12) as before with $\sigma_{1}$ and $\sigma_{2}$ replaced with $\sigma_{1}^{*}$ and $\sigma_{2}^{*}$. (a)

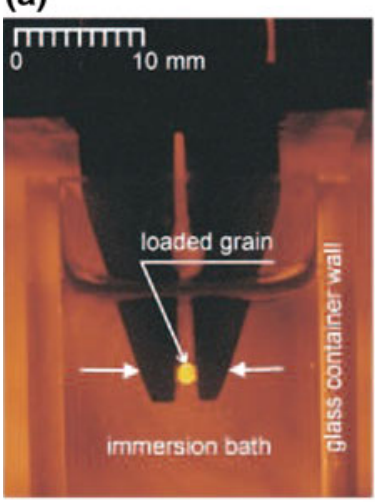

(b)

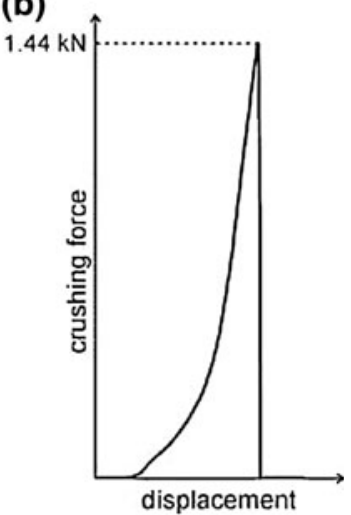

(c)

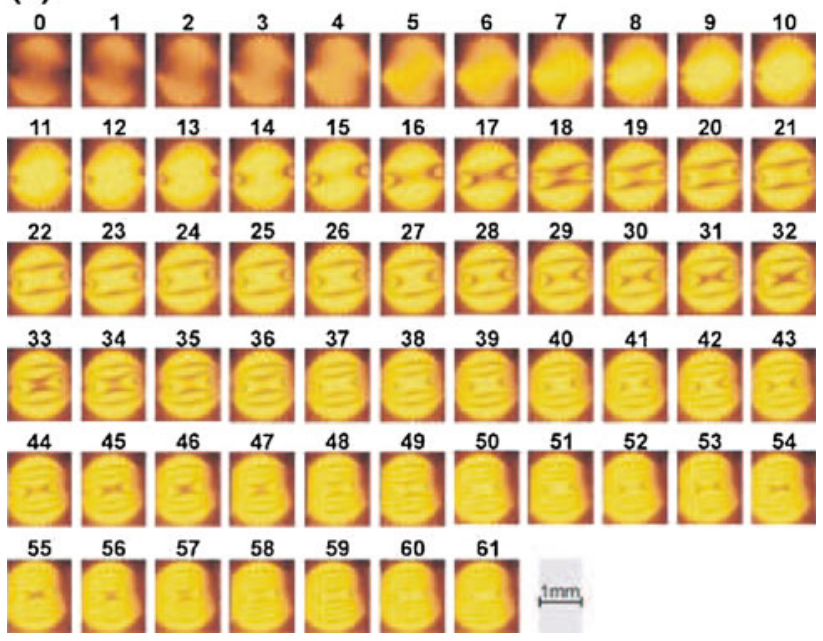

Fig. 10 Single spheroidal glass particles: a Loaded by steel jaws; c Viewed under polarised light; $\mathbf{b}$ Results of compression test 


\subsection{Particulate material}

We are concerned with the transmission of polarised light through a particulate material. We begin by repeating the experiment recorded in Fig. 7 with glass particles which are (nearly) spherical (Fig. 10) and with glass particles which have a more angular shape (Fig. 13), to check whether it is possible to observe photoelastic fringes in such small grains (having dimensions $\sim 1$ and $\sim 3 \mathrm{~mm}$ ). The spherical particles were made from glass having slightly different refraction index from the angular particles (1.52 and 1.47 respectively). The glass particles were immersed in a fluid with refractive index close to that of the glass, so that there was no refraction of light rays entering particles obliquely. It turns out to be rather easy to consult tables of refractive index in order to choose oils of correct refractive index-the choices are unlikely to be unique and local availability will in the end dictate which oil is used. The clove oil, used as an immersion liquid for the spherical particles (Starlite beads, both annealed and unannealed), was manufactured and supplied by Pollena Aroma in Warsaw, Poland. For the individual crushed Pyrex glass particles rizella oil was chosen; as only a small amount was necessary it was bought in a pharmacy in Gdansk. The success of the matching of the refractive indices is evident in the pictures that are presented. For both spherical and angular particles we can see the progressive formation and spread of fringes as the applied load is increased. Seven orders of exceptionally regular dark fringes were observed within the grain of Fig. 10 before it crushed. The value of crushing force estimated for seventh order dark fringes in $1.25 \mathrm{~mm}$ thick glass (grain diameter) should lie between 1.35 and $1.53 \mathrm{kN}$, if we assume values of photoelastic constants typical for glass [14]. The maximum force recorded during crushing tests on spherical grains was $1.44 \mathrm{kN}$. From this example we can see that the presence (or absence) of the dark fringes could be a good indicator of the local stress level in granular assemblies, provided that direct observation of individual grains were possible [21]. But recall that in this discussion we have ignored the fact that the observed fringes are a quasi-plane stress equivalent interpretation of the transmission of the polarised light through a three-dimensional particle under three-dimensional stresses.

There is a slight lack of symmetry observed in the images of the loaded single glass bead viewed using polarised light (Fig. 10). This arises because the Starlite beads are not perfectly spherical and because the loading configuration consists of rough jaws which are progressively closed but which are not precisely parallel. The particle is of the order of $1 \mathrm{~mm}$ diameter so that the handling and loading of the single particle are somewhat subtle. However, the purpose of showing Fig. 10 is to demonstrate that a particle subjected to an approximately diametral loading viewed under polarised light shows patterns which might be interpreted (as for the disc, Fig. 7) as successive fringes of stress difference in the specimen under test. However, the particle is in fact under a very non-uniform state of internal stress and the similarity of the patterns to those seen in really 2-dimensional assemblies (Fig. 7) could confuse the user into thinking erroneously that the bands in Fig. 10 also refer immediately to principal stress differences in the three-dimensional assemblies. It is this message that is important-the precise details of the loading of the single sphere are less relevant.

A visual feel for the complexity of the photoelastic patterns can be obtained by employing Matlab to convert original photoelastic intensity images into colour coded intensity images [19]. To obtain such an image, the original photoelastic picture in RGB colour separation has to be turned into a black and white image, based on 0-255 grey scale. The lowest possible intensity is then zero (black), and the highest 255 (white). A grey scale intensity image can then be plotted as a colour coded intensity map. A series of such maps, corresponding to the first nine load steps from Fig. 10 are shown in Fig. 11. Matlab can automatically detect the minimum and maximum intensities of the black and white image.

The values of the maximum intensities for all the loading stages applied to the spherical particle of Fig. 10 are plotted in Fig. 12 as a function of the corresponding load level. For the present purpose we are not concerned with the location of the maximum intensity within the grain: it is sufficient to know that the maximum intensity occurs for a certain load level at some point in the image, and that the intensities at any other points inside a grain can not be higher than this maximum. The graph in Fig. 12 presents rough data and obviously includes some statistical scatter (the statistical analysis of the intensity data is of minor significance for the present study). It can be seen that, after the first five load increments, there is a rapid increase of maximum intensity between 6 and 13 load increments. At about 15 load increments the value of maximum intensity stabilises at its ultimate value (with some statistical scatter). The first time that the ultimate value of maximum intensity is reached corresponds to the upper limit of the half fringe load range and at the same time to the upper limit of the monotonic relationship between intensity and load. A graph like that of Fig. 12 can thus help to define the experimental conditions under which a simple photoelastic response will be produced within a grain (central bright stripe), and thus a corresponding monotonic relation between load and intensity.

Crushed glass also exhibits dark fringes under increasing load; however, the fringes are much less regular. The grain in Fig. 13 shows fringes similar to the first and second order dark fringes observed in spherical grains. This would give an estimate of crushing force between 0.46 and $0.53 \mathrm{kN}$, which is substantially higher than the experimental 

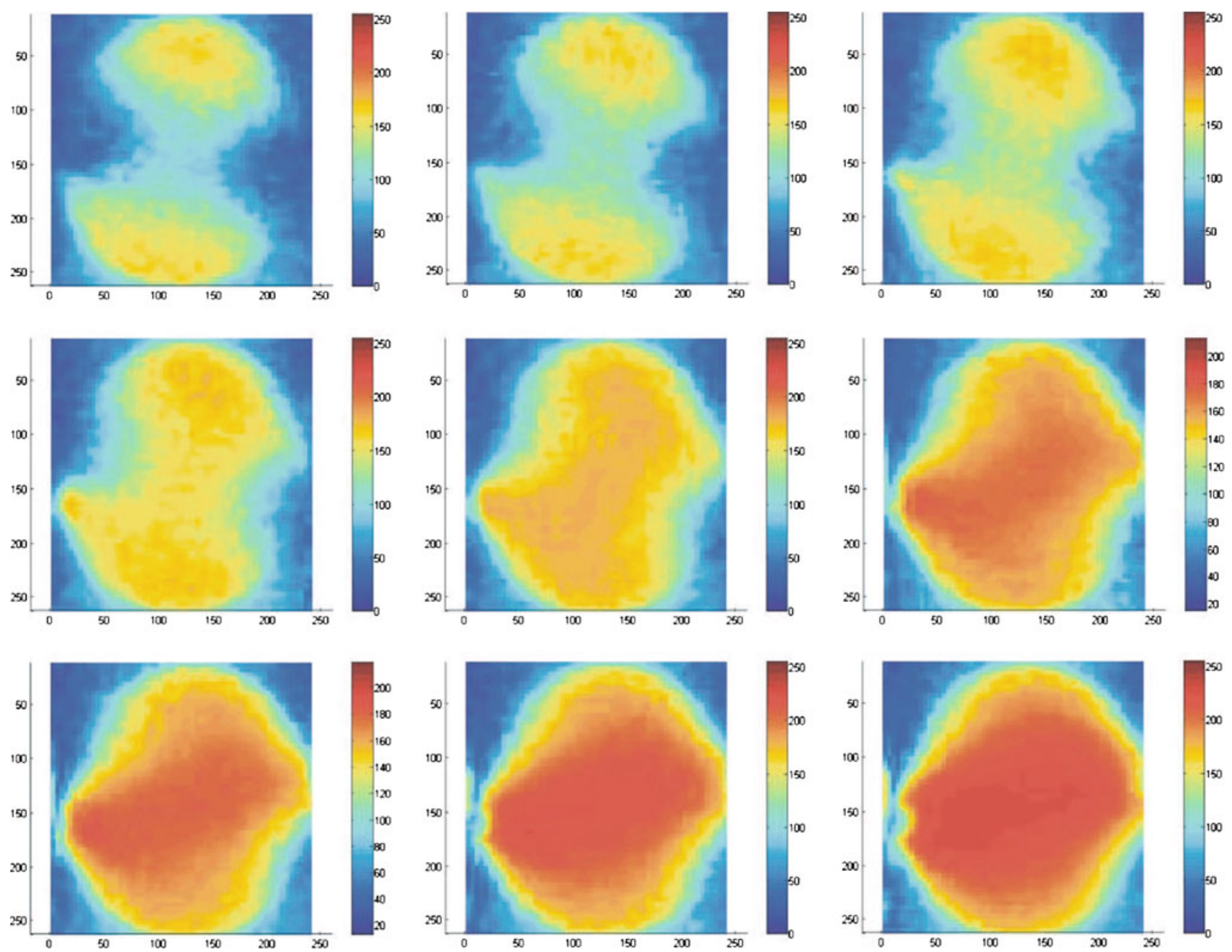

Fig. 11 Light intensity maps for images of single particle in Fig. 10, loading stages 1-9

average strength of crushed glass grains of $0.17 \mathrm{kN}$ (standard deviation $0.06 \mathrm{kN}$ ). But the grain shape is irregular and the state of internal stress quite different from the spherical particles.

The stress state in a single spherical particle loaded across a diameter is axially symmetric about this diametral axis of loading but a ray of light travelling through the loaded particle will encounter continuously varying three-dimensional stress states as it passes. We can compute the resulting effect by repeated application of (13) (Appendix) (Fig. 14), dividing the material through which the light ray passes into a sequence of infinitesimal slices each with a different secondary principal stress state and each with a different incremental effect on the polarisation of the light ray.

For the $j$ th element of the model of thickness $\delta z$ through which the light passes we need to know the orientation $\theta_{j}$ of the secondary principal stresses (3) and the relative retardation $\Delta_{j}=[2 C \pi \delta z / \lambda]\left(\sigma_{1}^{*}-\sigma_{2}^{*}\right)$. Then for this layer the transforming matrix becomes:

$$
\begin{aligned}
& \mathbb{M}_{j} \\
& \quad=\left[\begin{array}{cc}
\cos \Delta_{j} / 2-i \sin \Delta_{j} / 2 \cos 2 \theta_{j} & -i \sin \Delta_{j} / 2 \sin 2 \theta_{j} \\
i \sin \Delta_{j} / 2 \sin 2 \theta_{j} & \cos \Delta_{j} / 2+i \sin \Delta_{j} / 2 \cos 2 \theta_{j}
\end{array}\right]
\end{aligned}
$$

Each element of the material will produce a slightly different transformation $\mathbb{M}_{j}$ of the light passing through it, in a cumulative manner. The light emerging from the analyser is then the accumulation of all these successive transformations:

$$
\left(\begin{array}{c}
E_{x} \\
E_{y}
\end{array}\right)=\frac{1}{2}\left[\begin{array}{cc}
1 & -i \\
-i & 1
\end{array}\right] \times \prod_{j=1}^{N} \mathbb{M}_{j} \times\left[\begin{array}{ll}
1 & i \\
i & 1
\end{array}\right]\left(\begin{array}{l}
0 \\
1
\end{array}\right) k \exp i \omega t
$$

The stresses in a spherical particle of radius $R$ loaded diametrically parallel to the $x$ axis with a small but finite contact area defined by the angle $\alpha$ (Fig. 15) have been calculated by Russell and Muir Wood [20]. We want to analyse numerically the photoelastic effect for a ray passing through 


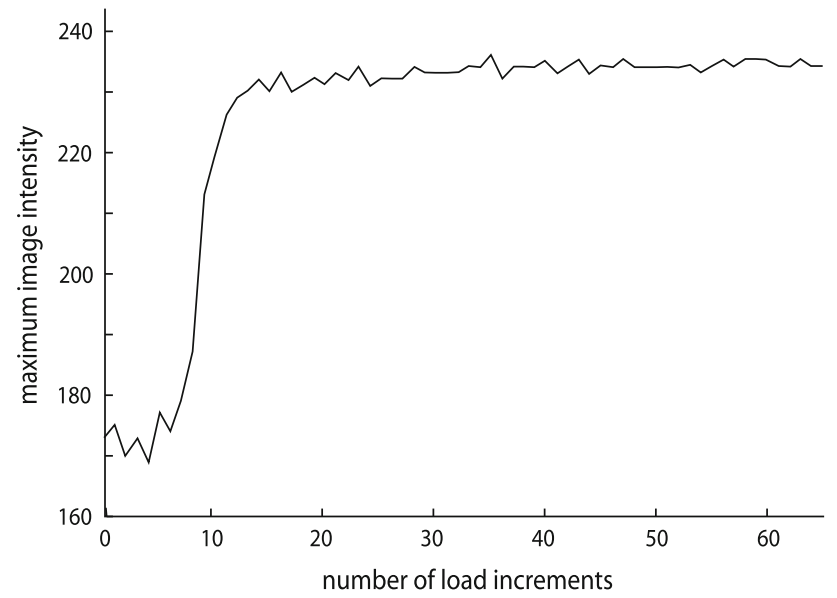

Fig. 12 Image intensity and particle loading for single spherical particle (Fig. 10)
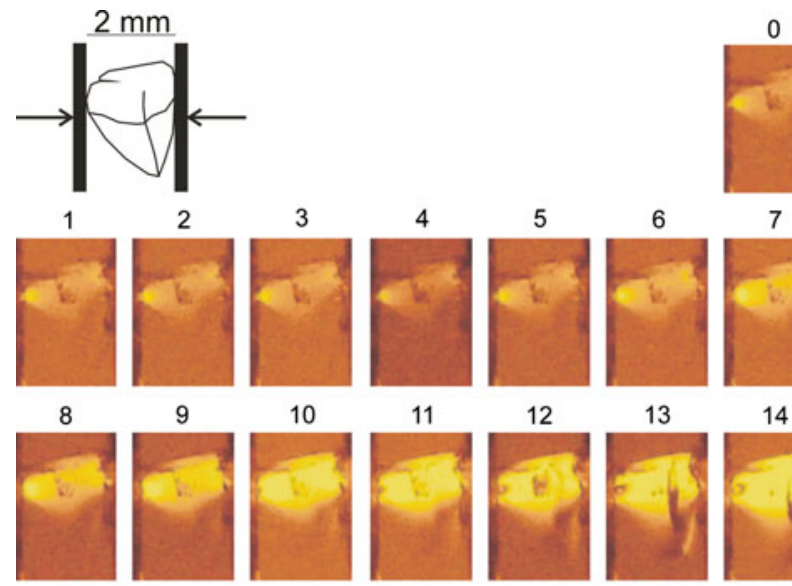

14

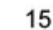

16
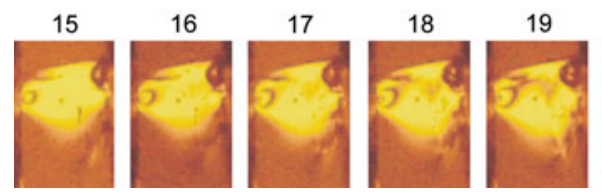

20

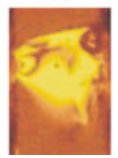

21

Fig. 13 Single particle of crushed glass under diametral loading

this particle. We are considering a sphere with its centre at $x=y=z=0$ and the light ray travelling in the $z$ direction. The sphere is loaded across a diameter coincident with the $x$ axis. We consider a cube of positive space of side $R$ (bounded by the planes $x=0, y=0, z=0, x=R, y=R, z=R$ ) containing one octant of the sphere. For points on an $x, y$ grid we can divide the cubical space occupied by a single particle of radius $R$ into $N$ elements in the $z$ direction. Given $x$ and $y$, the boundary of the sphere lies at $z_{s}=\sqrt{R^{2}-x^{2}-y^{2}}$ and for $z>z_{s}$ the light is passing through unstressed clove oil with no transformative effect. For $0<z<z_{s}$ we have an element of thickness $\delta z=R / N$ and spherical coordinates (Fig. 15) $\Omega=\tan ^{-1}(z / y), \Phi=$ $\tan ^{-1}\left[\sqrt{\left(z^{2}+y^{2}\right)} / x\right], r=\left[\sqrt{z^{2}+y^{2}}\right] / \sin \Omega$. We can calculate the stresses $\sigma_{x x}, \sigma_{y y}, \sigma_{z z}, \sigma_{y z}, \sigma_{z x}, \sigma_{x y}$. We are

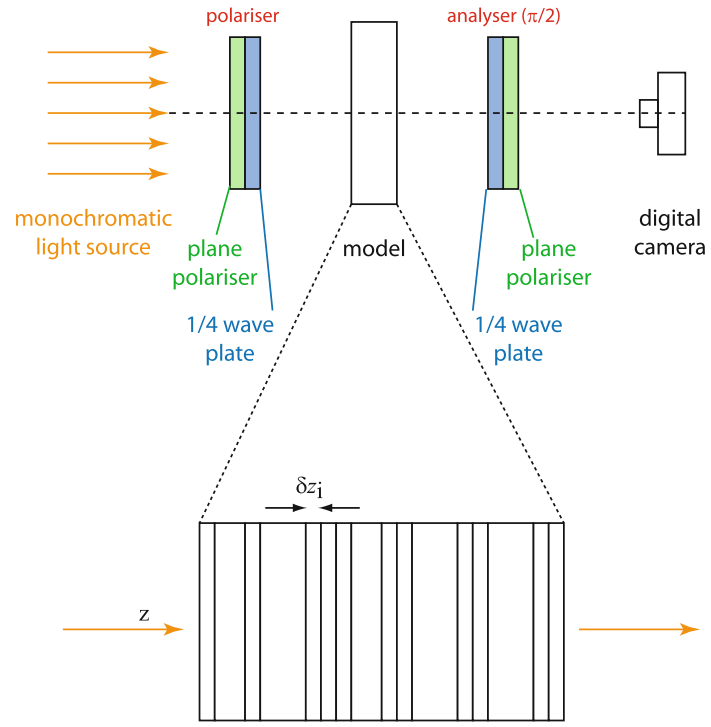

Fig. 14 Polariscope with model divided into infinitesimal layers

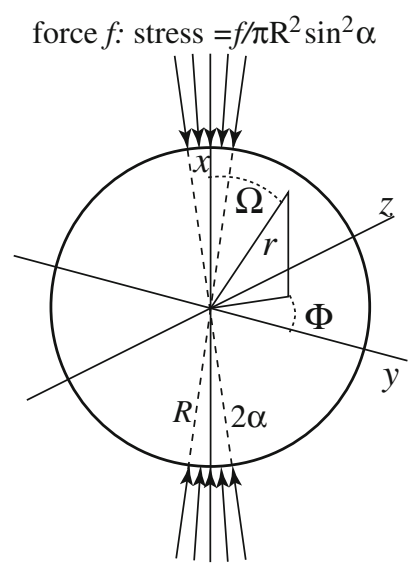

Fig. 15 Spherical particle under diametral loading

concerned only with the stresses in the $x, y$ plane orthogonal to the light ray and can compute the secondary principal stresses and their orientation in this plane. The orientation $\theta$ is given by:

$\tan 2 \theta=\frac{2 \sigma_{x y}}{\sigma_{x x}-\sigma_{y y}}$

and the principal stress difference is:

$\sigma_{1}^{*}-\sigma_{2}^{*}=2 \sqrt{\left[\sigma_{x y}^{2}+\left(\frac{\sigma_{x x}-\sigma_{y y}}{2}\right)^{2}\right]}$

For each element we can calculate $\theta$ and $\Delta$ and then form the matrix product to deduce the overall retardation, the orientation and ellipticity of the emerging polarised light (5). From symmetry, the light ray passes through the octant of the sphere twice, in opposite directions (the effects for $-R<z<0$ are the mirror image of those for $0<z<R$ ). 

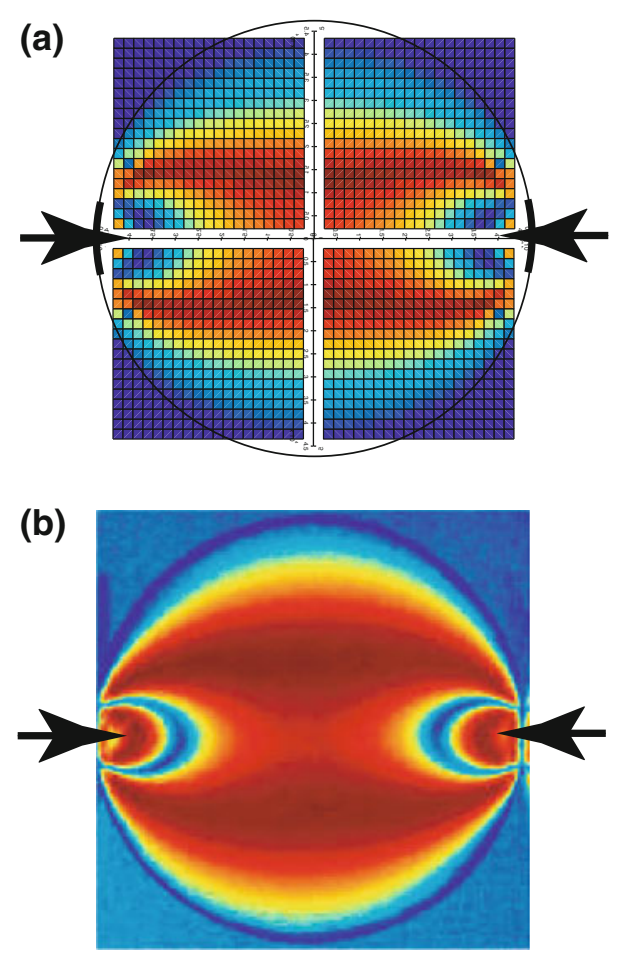

Fig. 16 a Calculated effect of passage of light through single particle under diametral loading; $\mathbf{b}$ Spheroidal particle under diametral loading (compare Fig. 10c)

We end with a distribution of the apparent principal stress difference for an equivalent layer of thickness $2 R$ under uniform stress. The result for the single sphere is shown in Fig. 16 where the colours indicate the resulting retardations. The correspondence with the photoelastic observations for the single spherical particle in Fig. 10 is apparent. Evidently the analysis ensures precise symmetry of the loading whereas, as noted, the experimental configuration slightly loses this ideal symmetry (Fig. 10).

The results of two-dimensional discrete element modelling (Fig. 4) show the very irregular nature of the network of contact forces which generates the internal stresses in the particles. And the results of three dimensional discrete element modelling confirm our expectation that, even for a configuration which has an overall plane strain constraint, every section through the model orthogonal to the direction of zero strain is different. The light ray thus passes through a sequence of particles and surrounding fluid (Fig. 17), each particle loaded differently and in a different orientation relative to the ray, and each particle having a complex internal stress state resulting from the particular combination of contacts with adjacent particles to which it is subjected.

We could in principle repeat the procedure used for the single sphere and calculate the cumulative effect of passing a light ray through the entire assembly. Of course for the actual assembly of particles the stress state in any single particle will

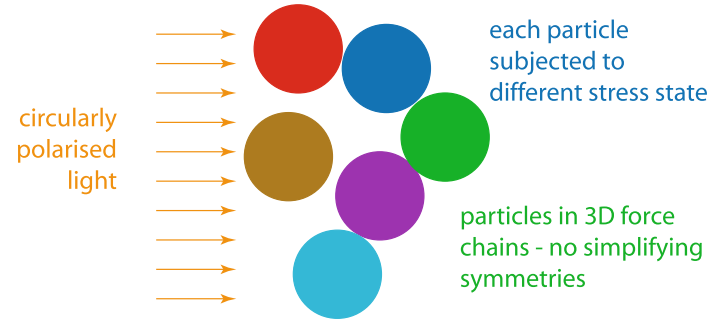

Fig. 17 Light and particles

be much more complex than that shown in Fig. 16 because any one particle will in general experience multiple contacts with different contact forces in different orientations. Given the nature of the granular material (Fig. 17) this cumulative effect would be most readily calculated numerically but the calculation for a fully three-dimensional particulate assembly is beyond the scope of this paper. The analysis of the passage of light through a single loaded sphere provides an illustration of the typical building block of the numerical process.

However, given the form of (5), we can propose that the eventual result will be equivalent to the product of a large number of random matrices. Within any single particle there will be some continuous correlation between the stress state in successive infinitesimal elements through which the light ray passes. However, there will be no correlation between the stress states in successive particles: hence the deduced randomness. Typical results show that such repeated multiplication ends with an asymptotic matrix which lacks information (e.g. [27]).

\section{Model tests}

The material being used here consists of Starlite glass ballotini (Fig. 9) which can be seen to be more or less spherical and more or less single sized. As supplied these particles are not completely free of internal stresses as a consequence of the manufacturing process - the rapid cooling of the glass leaves the outside of the particle in a state of tension. It is necessary to anneal the particles-heating them up to a temperature of $500^{\circ} \mathrm{C}$ for a period of $2 \mathrm{~h}$ and then allowing them to cool extremely slowly. The glass of the particles has a refractive index of 1.524. In order that the assembly of particles should be perfectly transparent to the incident light it is necessary to immerse the glass particles in a fluid having the same refractive index (according to the principle of integrated photoelasticity). As noted above, clove oil proves to be ideal for this purpose. The light ray is then able to pass freely and without significant refractive deviation through the particle assembly. 


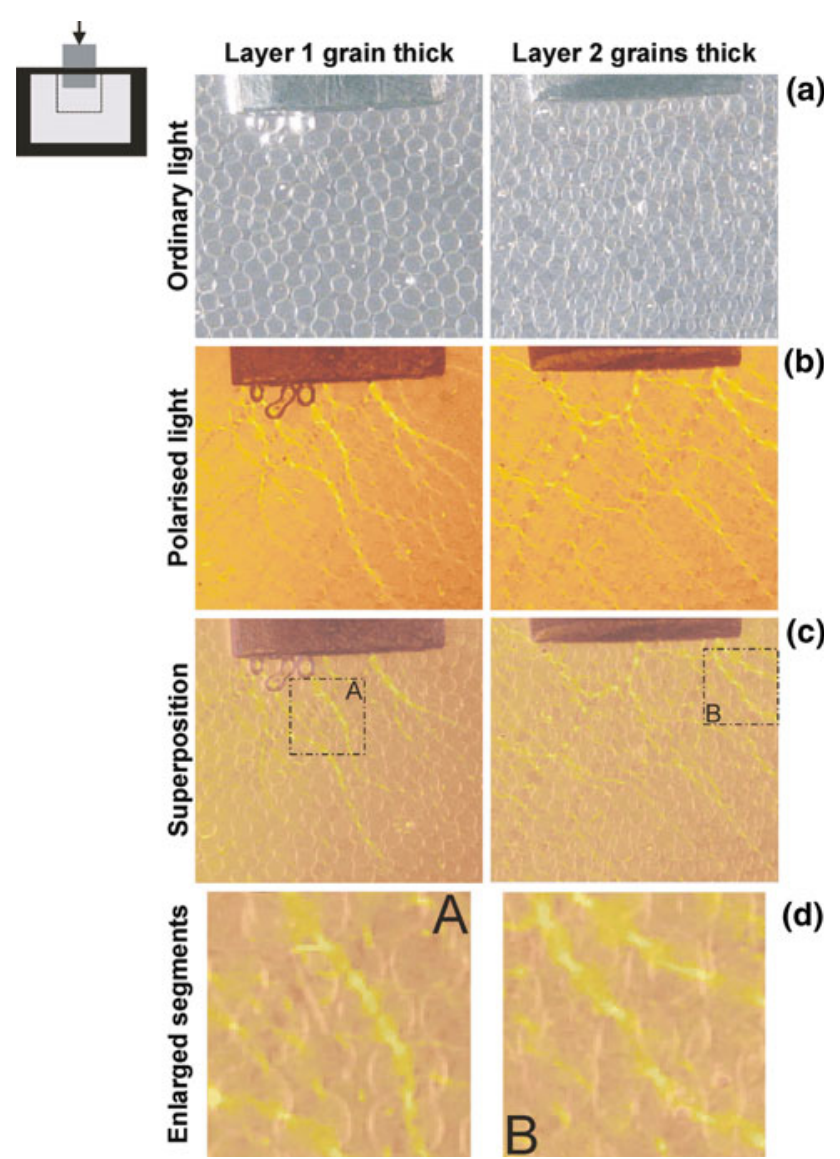

Fig. 18 a Particle boxes 1.4 and $2.6 \mathrm{~mm}$ thick containing approximately one and two layers of particles respectively; $\mathbf{b}$ Particles viewed under polarised light; $\mathbf{c}$ Superposition of polarised and unpolarised images (a) and (b); d Enlargement of particles under load

A series of simple test boxes have been made with dimensions $20 \times 30 \mathrm{~mm}$ and thicknesses 1.4 and $2.6 \mathrm{~mm}$. The thinnest of these boxes can only contain a single layer of particles. The $2.6 \mathrm{~mm}$ box can contain two layers of particles-though of course the particles do not actually sit in independent layers. In each case, the particles are deposited in the box and then saturated with oil, with care being taken to ensure that no air voids remain. A rudimentary 'footing' has been used to load the particles simply to confirm that in these almost two-dimensional assemblies the force chains through the spheres (not discs)(Fig. 18) correspond qualitatively with those anticipated from numerical analysis (Fig. 4). The images shown here were taken in circularly polarised yellow sodium light. Saturation with clove oil in this test was not quite perfect: a few air bubbles are visible under the left side of the 'footing'.

The top pair of photographs (Fig. 18a) show the particulate material seen with white light (unpolarised). The individual particles can be seen, and the overlapping of particles in the thicker box (right-hand image) is very clear. The pair of photographs (Fig. 18b) show the same assembly seen with circularly polarised light and the third pair (Fig. 18c) superimposes the images from (a) and (b) to show more clearly the relationship of the features that are seen to the particles themselves. Finally, the pair of images (Fig. 18d) shows a close-up view of part of the superimposed images (Fig. 18c).

The eye is drawn to the bright regions in images (Fig. 18b, c). These can be seen to link particle contacts and to be of a scale which is smaller than the individual particles. The enlarged images (d) reveal a hint of patterns within individual particles which are, as expected, similar to those seen for the single sphere (Fig. 10, images 6 and 7, for example). We note that many particles in the assembly are carrying no or almost no load. Close observation also reveals some dark bands, linking particles which are less heavily loaded so that the bright region appears only local to the contacts with neighbouring particles. There may also be shear stress transmitted through some contacts which will distort the distribution of stresses within the particle and hence the nature of the resulting photoelastic effects near particle contacts.

The second (right-hand) set of pictures in Fig. 18 relate to a sample which is $2.6 \mathrm{~mm}$ thick. The particles no longer lie in plane layers but the sample is thin enough that all particles can still be seen. Superimposing the normal white light view of the particles onto the polarised image reveals bright paths passing through the heavily loaded contacts, with darkness in the less heavily loaded particles. But the result is now dependent on the three dimensional nature of the material. The force chains do not stay in one plane but dodge to and fro depending on the positions of the particles and their contacts. While similar qualitative interpretative statements can be made, it is now less obvious exactly how the bright regions are associated with individual particles and certainly not with any particular layer within the material. Even though the models are roughly two dimensional, the glass particles seem to be diametrally loaded - the internal photoelastic effects observed within individual grains forming the force chains resemble the early stages of the test shown in Fig. 10.

The tests reported by Leśniewska and Muir Wood [15] looked at various aspects of the response of a special plane strain model test in which Starlite glass balls were subjected to loads and deformations in a box of thickness $20 \mathrm{~mm}$. In such a box there are many 'layers' of particles which are expected to interact to produce the overall response, although no individual particle will feel itself constrained to sit in any one layer parallel with the plane of straining and will interact with particles in adjacent 'layers' and may well even move into an adjacent layer as the mass of particles is deformed.

The glass particles are pluviated into the box and then saturated with oil. The dramatic effect of immersing the particles in a fluid having the same refractive index can be seen in Fig. 20. The granular material is loaded by a somewhat articulated surface footing (Fig. 19) the segments of which 


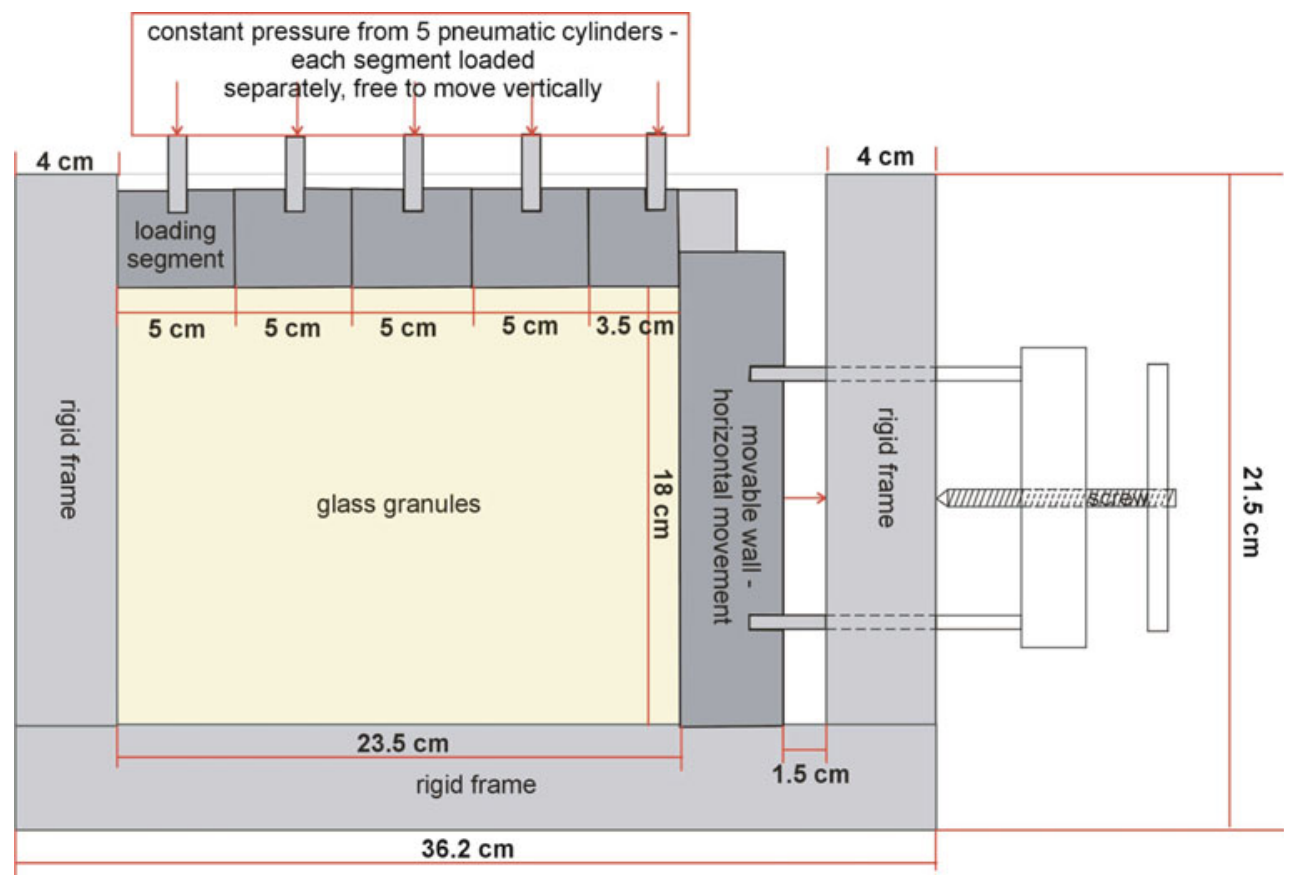

Fig. 19 Box used for loading of glass particles of width $20 \mathrm{~mm}$

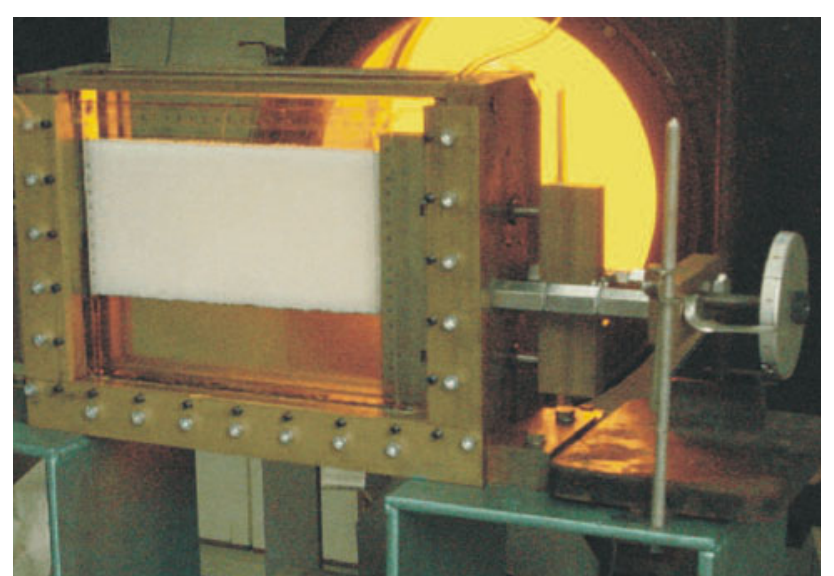

Fig. 20 Assembly of glass particles being saturated with clove oil

are loaded individually to guarantee an adequate stress level for birefringence to be seen. The granular material is contained by a movable 'wall' which is supported at two levels. Once the surface load has reached the chosen value, the wall supports are released in a series of small displacement steps so that the wall itself translates and rotates under the pressure transmitted through the mass of glass particles.

The sodium lamp provides approximately uniform illumination over a roughly circular area which covers the area of the apparatus of interest. The dark areas to the top and bottom left of Fig. 21 are thus areas of low illumination.

First we look at a single image (Figs. 21, 22). This sample was loaded at the surface in steps of $0.2-3.2 \mathrm{MPa}$ and then unloaded to 1.6 MPa. The 'wall' was released in steps of $0.0625 \mathrm{~mm}$. This image comes after the 8th displacement of the wall. Seen as an overall image the presence of 'light stripes' is very evident. However, when one looks in detail at patches of the overall image some of the texture of these bright regions can be detected. Our observations of single and double layer samples (Fig. 18) now lead us to understand that the observation of a bright region in the thick sample will be the consequence of strongly loaded particles existing somewhere through the thickness of the sample. These particles are responding to the contact forces in their vicinity. There is an impression from two-dimensional discrete element analysis that the strong force chains are somewhat aligned in the direction of the major principal stress but the DEM results in Fig. 4 indicate that the force chains are not locally straight.

The smaller images in Figs. 21 and 22 show detail of the complete image. The texture is much finer than the size of individual particles and even in regions of rather low stress there is a hint of some preferred directions to the texture which are visually indicated as much by the bright stripes as by dark stripes. Dark stripes are somewhat visible in the single layer of particles (Fig. 18) and possibly indicate chains of particles which are actually transmitting significant shear at the contact so that the axes of symmetry of the stress state within the particle are not aligned across the loaded diameter.

Second we look at a pair of successive images (Fig. 23). Besides other features they show a faint, brighter, wedge shaped area in the top right corner of the image, which is slightly more distinct in the second image. The existence of a 'rigid' wedge-shaped block of the granular material, a sort 


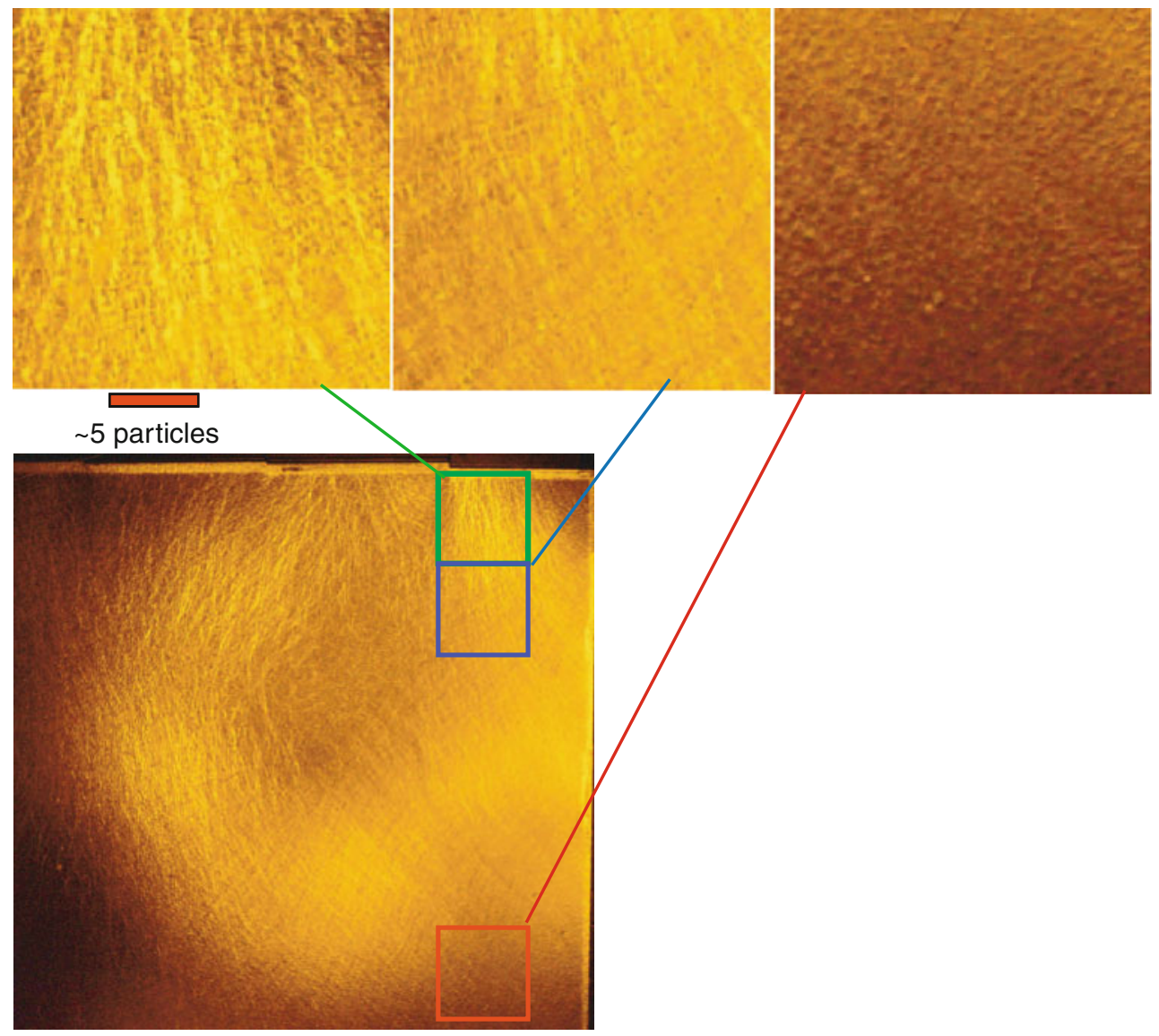

Fig. 21 Detail of polarised light image—regions near 'wall'

of Coulomb wedge, is not unexpected for an experimental scheme which develops active soil pressure on an inflexible retaining wall. Figure 23 shows that the rigidity of the wedge comes from the increased number of highly ordered force chains within its volume. The chains are slightly arched and somewhat parallel to the wedge boundary. They join together the bottom of the first loading segment and the back of the 'wall'.

The amount of visual information included in an original photoelastic image of a large 3D granular assembly far exceeds the information which is contained in a typical example of continuum photoelasticity (for example, a structural model) or in an image representing a small, one layer granular sample. Different methods of analysis, suitable for managing large amounts of visual data, have to be developed. Such methods should allow the elimination of unimportant information without the simultaneous loss of significant data. Whether particular features are important or not depends on the phenomenon being studied.

The simplest way to visualise changes introduced into photoelastic images by any variation of boundary conditions is to subtract one image from the other. The images exist as matrices of image intensity values (RGB format) and it is straightforward to perform arithmetic operations on them. The images of Fig. 23 are separated by a small movement of the wall of $0.0625 \mathrm{~mm}$ which leads primarily to a rotation about the toe of the wall with most of the displacement occurring near the top, near the loaded articulated surface. To the naked eye the images look more or less identical. Thus Fig. 24a shows the effect of subtracting the second image from the previous image, pixel by pixel. The differences are enhanced in order to improve the visualisation. The colour coding generated by MATLAB indicates areas where the brightness is higher in the first image by comparison with the second. There is a bright crescent-shaped region extending downwards from the edge of the first 'footing' (Fig. 19). The interpretation must be that in this region some of the particles through the thickness of the model are either leaving strong force networks or, although staying in a network, subjected to lower contact forces. Figure 24b shows the complementary effect of subtracting the first image from the subsequent image, pixel by pixel. Now there is a bright region near the top of the 'wall' (Fig. 19) and also a less bright region extending back into the model from the edge of the first footing. The interpretation must be that in these regions some of the particles through the thickness of the model are 


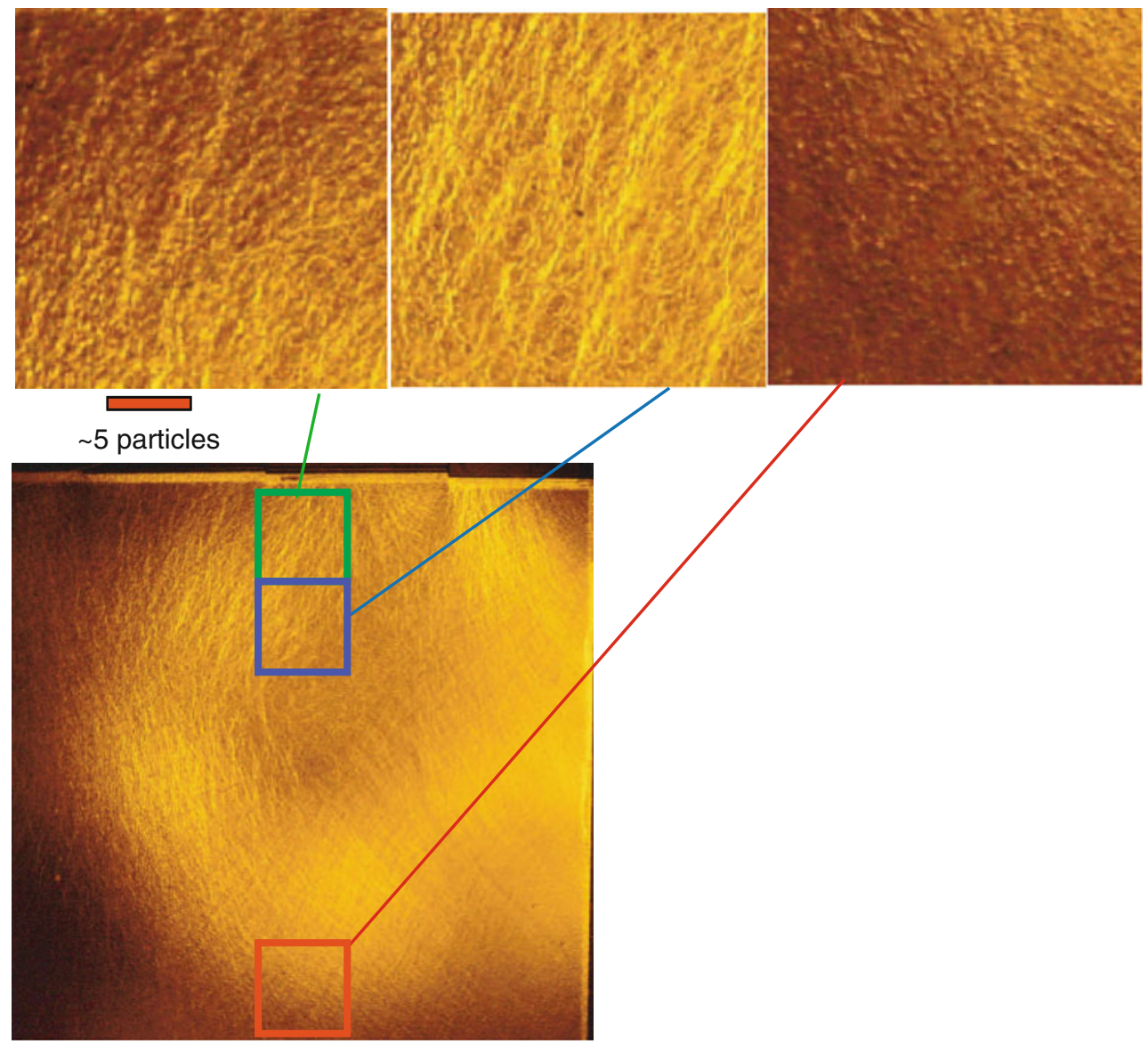

Fig. 22 Detail of polarised light image—regions away from 'wall'

Fig. 23 Polarised light images (a) before and (b) after small displacement of 'wall'

\section{(a)}

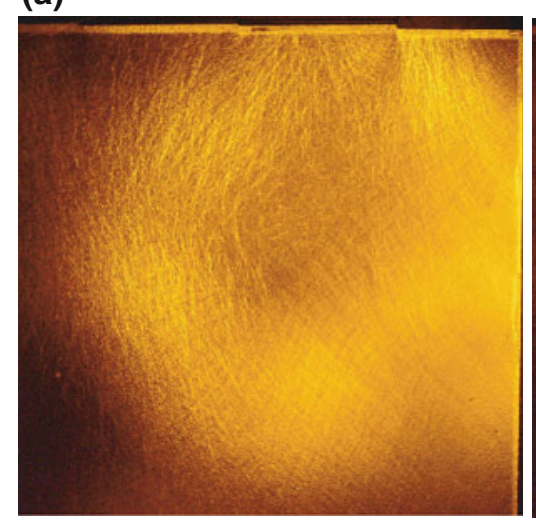

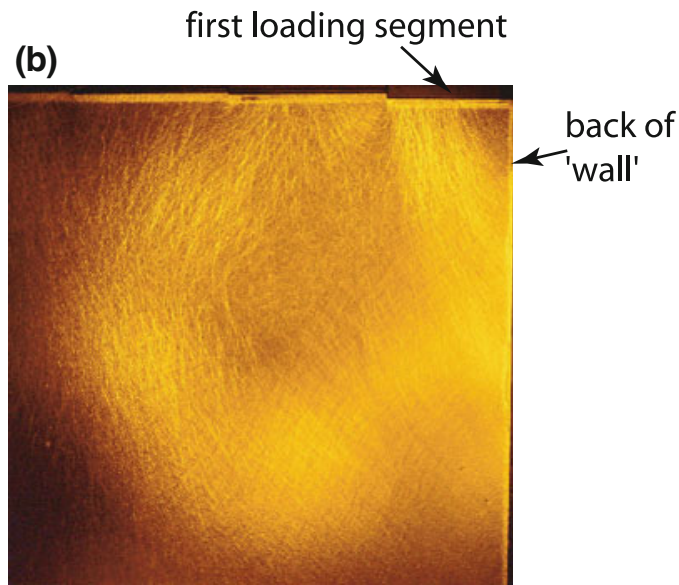

either joining strong force networks or, already in a network, being subjected to higher contact forces.

The same brighter wedge as in Fig. 23 can be (even more easily) recognised after the subtraction. Its appearance in Fig. 24b means an increase in brightness of the force chains located within the wedge, after the wall movement (positive result of subtraction). This suggests that the wedge became more rigid after the displacement (leading to increased loading of force chains). If so, no significant deformation should be expected within its volume. The bright crescent-shaped area visible in Fig. 24a represents corresponding weakening of the granular material (unloading of force chains). It would be expected then that significant deformation would be concentrated in this area. The correctness 
(a)

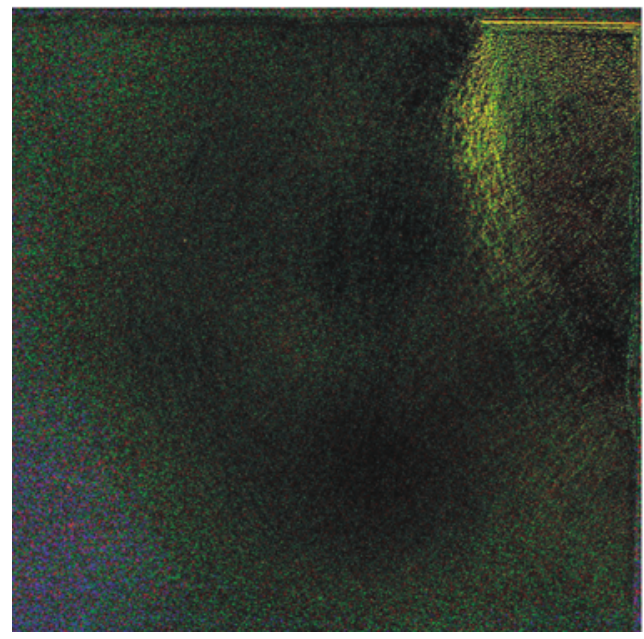

(b)

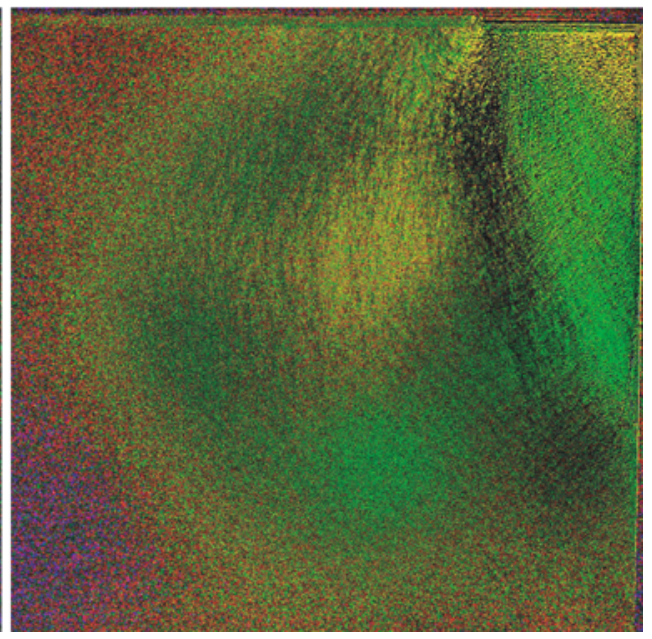

Fig. 24 a Subtraction of next image from previous image; b Subtraction of previous image from next image (Fig. 23)
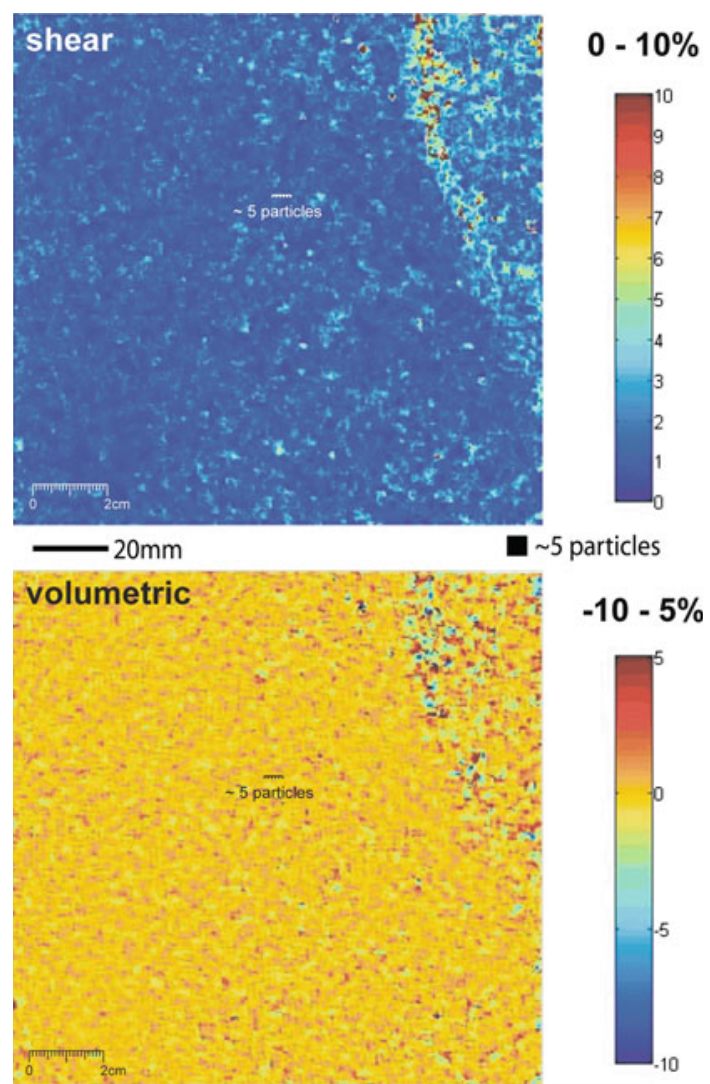

Fig. 25 Contours of calculated shear strain and volumetric strain for loading increment matching the two polarised images in Fig. 23

of such a reasoning can be confirmed by comparing these subtracted photoelastic images with the relevant strain fields obtained by other independent methods. The most suitable seems to be digital image correlation (DIC).

\section{Digital image correlation}

Even when viewed under white light the virtually transparent particulate material retains sufficient visual contrast and texture for successive photographs to be compared to detect relative movements within the material. The techniques of Particle Image Velocimetry [29] or Digital Image Correlation [12] can be used to determine displacements and hence fields of strain. Our interest here is to compare some aspects of the texture of these strain fields with the texture seen in the images taken with polarised light. The texture now is an effect of the transmission of the unpolarised white light through the entire $20 \mathrm{~mm}$ thickness of the sample and it is not possible to identify individual particles.

Determination of fields of strain by comparison of successive images taken with normal white light has been achieved using the program GeoPIV [28]. The discussion of the necessary numerical checks to ensure that the results are valid is contained in that paper and also in Leśniewska and Muir Wood [15]. Two sets of results are shown here. First, Fig. 25 shows maps of incremental shear and volumetric strain for the increment of wall displacement corresponding to the pair of polarised light images in Fig. 23. These maps have been calculated from displacement fields obtained using a $32 \times 32$ pixel correlation window on an $8 \times 8$ grid. The average particle diameter is about 13 pixels. The strains are concentrated in regions which define some sort of mechanism between the 'footing' and the 'wall'. The strain concentrations (both shear and volumetric) are situated along the wedge of Fig. 24b; however, there is no continuity in either plot. They seem to coincide roughly with the bright crescent of Fig. 24a, as expected. This confirms the interpretation proposed for the subtracted images in the previous section. The volumetric strains fluctuate around zero with regions of expansion 
Fig. 26 Comparison of textures of polarised light images and calculated strains for region in top corner of model beside wall (a) shear strains

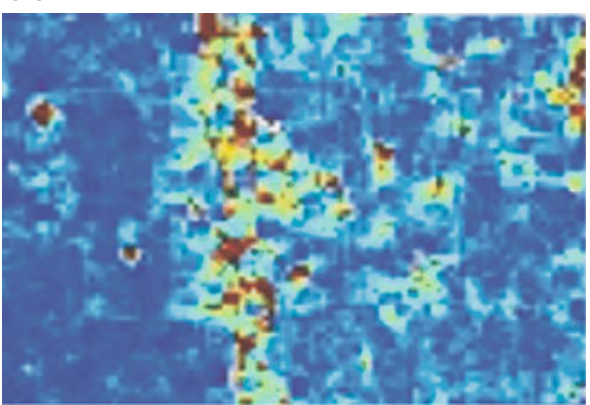

5 particles

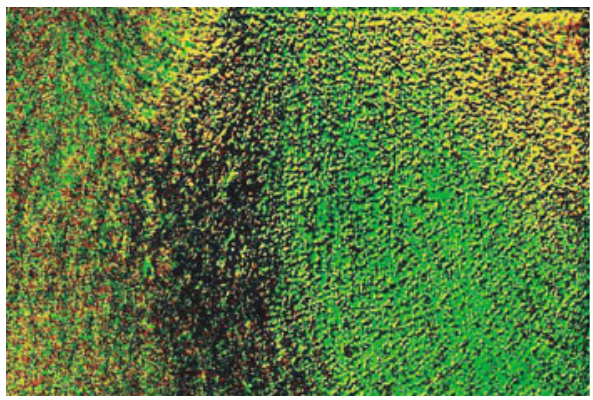

(c) next minus previous (b) volumetric strains
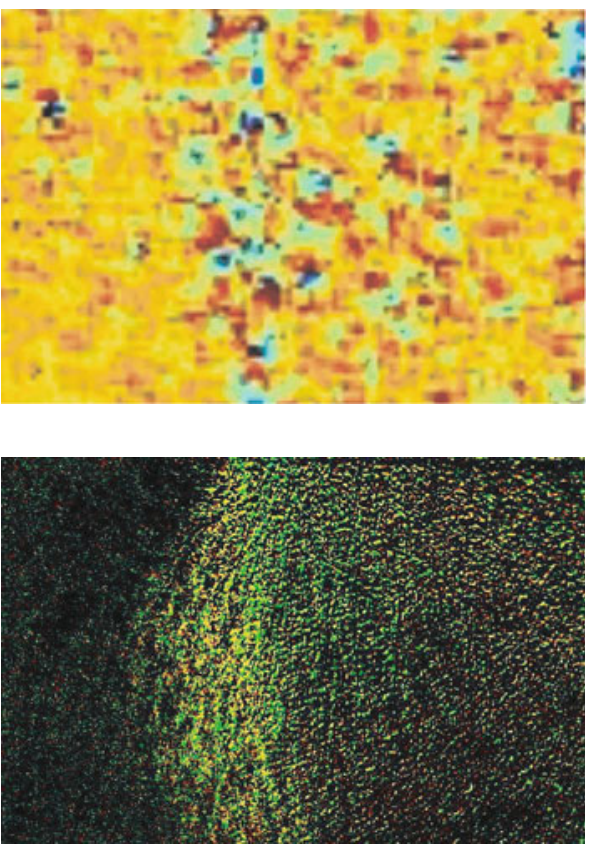

(d) previous minus next alternating with regions of compression. The scale of these fluctuations is of the order of 2-3 particles, as seen more clearly in Fig. 26 which shows an expanded view of a small region of the shear strain and volumetric strain fields near the top of the wall. This scale matches the length scale of the strain patterning seen in tests reported by Hall et al. [13] using Schneebeli rods as a two-dimensional analogue of a granular material and performed in the Grenoble $1 \gamma 2 \epsilon$ apparatus which is able to impose a general system of controlled normal and shear strains to a plane assembly of the rods. The rods were also somewhat (but a little less) single-sized.

Figure 26 also shows the expanded views of the polarised light images of Fig. 24a, b. There is texture in both sets of detailed views but the scale of the texture of the polarised light images is of sub-particle level.

\section{External stress level}

We have seen that the nature of the visible photoelastic effects inside a single grain depends strongly on the level of the load applied. The effect can be geometrically simple, taking the form of a bright elongated central stripe, which clearly indicates the direction of particle loading (Fig. 7, images 11-15, Fig. 10, images 7-11); or it can be quite complex, as a result of the multiple and often irregular dark fringes that appear at higher loads (Figs. 7, 10, 13). In large, multilayer granular assemblies, it is not possible to trace the response of individual grains to the applied boundary stresses, and the overall picture will benefit if it represents the amplification of the simple photoelastic effect in those individual grains. However, even in small 2D samples grains are not uniformly loaded, as Fig. 2 shows, and each grain exhibits the fringe pattern characteristic of the different stages of its potential loading history.

The same procedure can be applied to granular assemblies. Fig. 27b (column A) shows photoelastic images representing the early stages of the test shown in Fig. 21. The images are cut off from the whole field image - they cover the area indicated in Fig. 27a. The test begins with the steady increase of the loading applied to the individual articulated surface 'footings' (Fig. 27) before the 'wall' is allowed to move laterally (Fig. 21). A visual inspection of the images shows that bright stripes form from the first loading step $(0.2 \mathrm{MPa})$ and become more distinct with increasing load. The width of the bright stripes does not exceed the average grain diameter and grows with applied load. There is an impression that both the brightness and the number of the stripes increase with the applied load. However, we do not know the position of the bright stripe within the granular assembly - the photoelastic images of Figs. 21 and 22 are a mere projection of 3D features on a plane-so we cannot be sure whether we observe a single force chain (which seems unlikely) or a visual product of several superimposed chains. The fact that the width of the observed bright stripes does not exceed the average grain diameter, even for loads higher than those indicated in 
Fig. 27 Image intensity for wall test (Fig. 21)

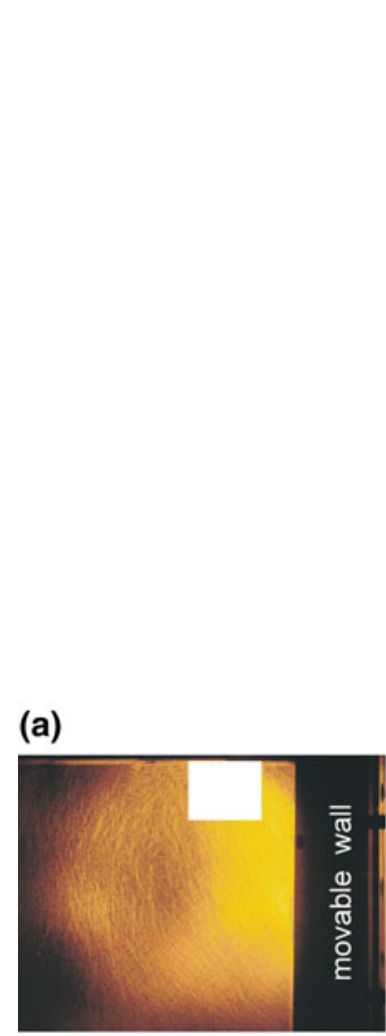

(b)

A
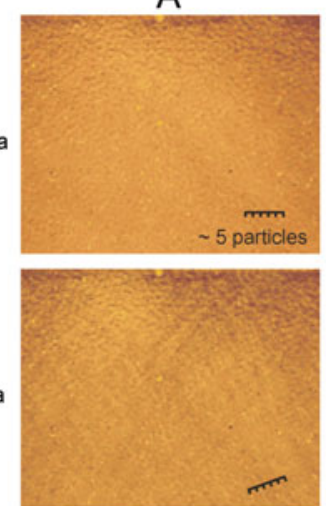

$0.2 \mathrm{MPa}$

$$
\text { (5) }
$$

$0.4 \mathrm{MPa}$

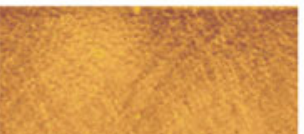

$0.6 \mathrm{MPa}$
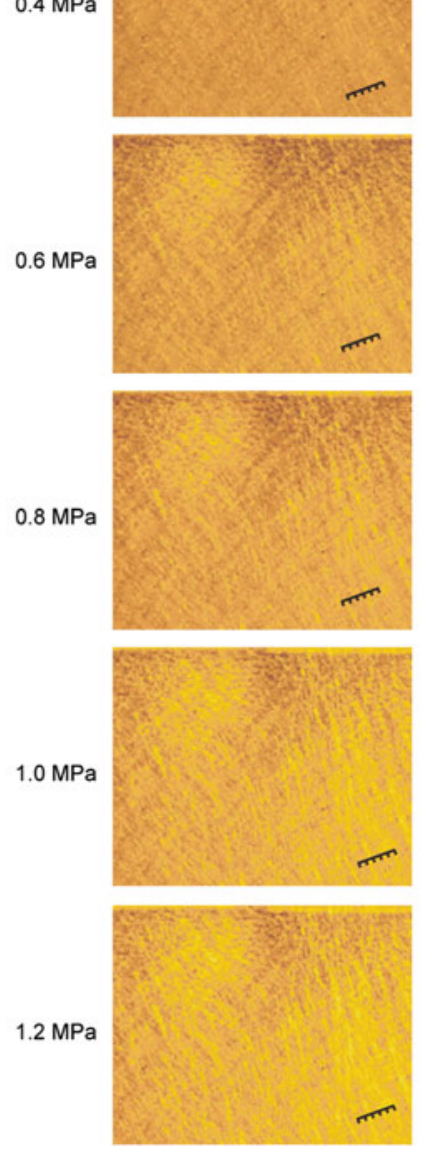

B
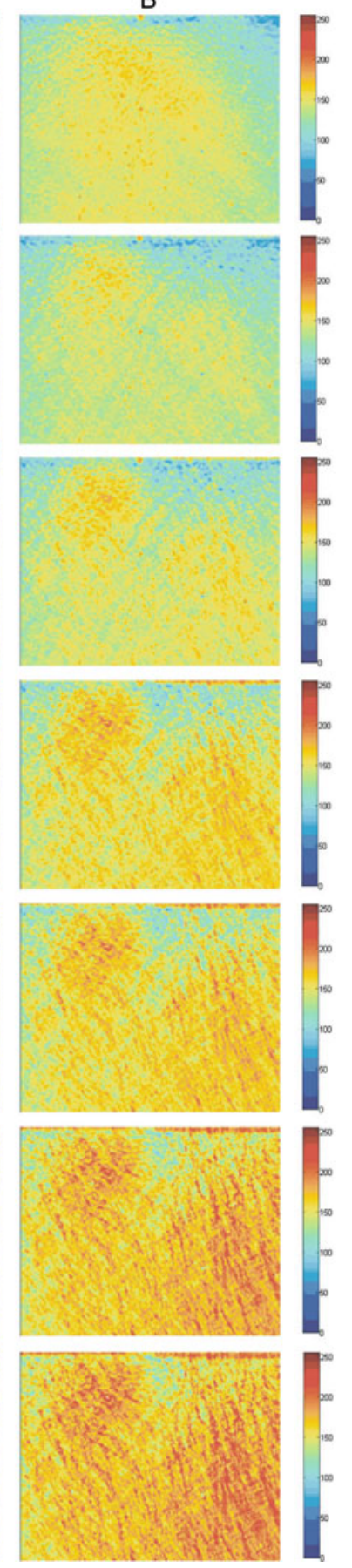

Fig. 28, suggests that they can be built of monochromatic fringes within individual particles, corresponding roughly to images 4-10 in Fig. 10.

Turning the photoelastic images into colour coded intensity maps (Fig. 27b, column B) allows us to inspect the character of the relation between maximum image intensity at each step of the test, and the external load applied to the articulated surface footings. The result is shown in Fig. 28, which covers the full range of the external loading tested (0-3.2 MPa). The curve thus obtained for the large granular assembly has a similar character to that presented in Fig. 12 for a single spherical grain. The initial intensity drop in Fig. 28 can be explained by possible grain rearrangement at the beginning of the test.

There is an approximately linear part of the intensity curve between 0.4 and 1.0 MPa of external load-the load 


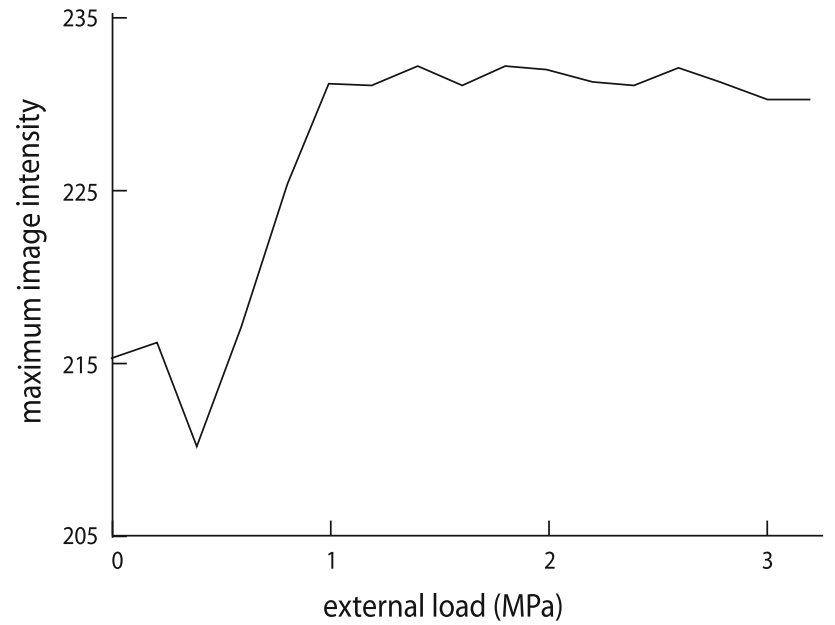

Fig. 28 Image intensity and loading stage for wall test

range within which the maximum intensity related to the applied load does not exceed its ultimate value in any point of the granular sample. The load range $0.4-1.0 \mathrm{MPa}$ roughly defines the range of monotonic relation between image intensity and load. There is then an approximately constant ultimate value of maximum image intensity (about 231 on the 0-255 scale) (Fig. 28) above 1.0 MPa of external load. In other words, there is at least one point (pixel) within the area under inspection where this ultimate value of image intensity is reached. Consequently we can expect a nonmonotonic (sinusoidal, above half fringe order) relationship between the light intensity and the applied load at at least some points of the sample in this load range. Dark fringes can appear within some grains, which are not easy to detect for the complete assembly, but which possibly influence local values of image intensity. It suggests that no quantitative photoelastic measurements based on image intensity should be performed outside the load range of the linear (or at least the monotonic) part of the intensity-load function.

This also seems to be important if arithmetic operations are to be performed on digital intensity images. For example, Voloshin and Berger [25] used 16 exposures of the same photoelastic field to obtain an average result with reduced random noise; Haake and Patterson [11] used a large number of scans and subtracted the dark noise from subsequent intensity readings; Geng et al. [9] used a series of images of 2D assemblies in order to obtain an average response and also subtracted successive images to remove the linear hydrostatic head effects. Figure 24a, b of this paper also show an example of subtracting images, in order to obtain an incremental photoelastic response to varying boundary conditions. A systematic study of the meaning and applicability of arithmetic operations on photoelastic images is still lacking.

\section{Conclusions}

Some simple non-standard post-processing procedures are also demonstrated for photoelastic images, making use of the Matlab Image Processing Toolbox. These are intended to help in extracting as much 'hidden' information as possible from photoelastic images. The response of photoelastic materials such as glass to varying test loading conditions is an example of such hidden information: the material is relatively insensitive (in terms of the link between change in stress and change in image intensity) so that small changes in some regions are easily obscured by more stable parts of the photoelastic image.

For a two-dimensional continuous stressed material, loaded in plane strain, such as the disc in Fig. 7, the image conveys information about a continuous stress field which is uniform through the thickness of the model. Concepts such as principal stresses and principal axes of stress have a clear and certain meaning. The dark regions appear (as multiples of principal stress difference) in sequence as the load is increased. The intensity of the transmitted light does not vary monotonically with the principal stress difference. Arithmetic operations - such as addition or subtraction - on the intensity of the light recorded in successive pairs of such images have no particular significance since an increase in intensity may indicate either an increase or decrease in the principal stress difference (Fig. 6).

However, the images of the three-dimensional particle assembly do not relate to a continuous uniform stress field but to an integrated effect of varying stress states through the various particles. Individual particles within a granular assembly will be subjected to very different internal stress states as the boundary stresses are varied. Within any one loaded particle there will, if the stress level is not too high, be a central region of enhanced light intensity which may be somewhat parallel to the global direction of major compressive principal stress. However, the photoelastic observation for the complete assembly is a three-dimensional averaging of information through the thickness of the sample. Numerical analyses using Discrete Element Methods quantify the variations of the force chains through $3 \mathrm{D}$ assemblies and could be used to compute the expected effect of passage of polarised light, extending the procedure used here to compute the apparent retardation produced by a single spherical particle (Fig. 16).

Large 3D granular assemblies require methods of analysis different from standard methods of continuum photoelasticity. There exist fully observable photoelastic effects even within very small individual grains - this effect can spoil the results of any macro scale photoelastic measurements to an unknown extent. It may be possible to control the photoelastic effect in individual grains simply by controlling maximum image intensity and adjusting the external load so that it does 
not exceed the maximum level for the monotonic relationship between stress difference and photoelastic output. Full-field observation supported by the Matlab Image Processing Toolbox makes such control possible. A simple procedure for verifying the conditions of photoelastic model tests based on maximum intensity check has been proposed in this paper.

Model tests presented in this paper seem to confirm the accepted interpretation of the existence of a relation between the orientation of the 'light stripes' and the principal stress directions. There has been no attempt made in this paper to determine the latter, either experimentally or theoretically, partly because more experimental data are necessary to study the problem over a wider range of numbers of grains included in the granular sample (and also over a range of ratios of loading dimension to grain diameter), and partly because it would be difficult to use continuous mechanical or photoelastic theoretical concepts to study a sample containing either a limited number of grains (there is a problem of definition of stress) or a large number of grains (because of the possible problems in interpreting the photoelastic data). The principal stress directions at the loaded boundaries of the sample are usually known, however, so it is possible to look for a local relation between them and the 'light stripes' visible in the same region. Visual inspection of Fig. 18, for example, suggests that the arrangement of the grains significantly affects the direction of the force chains for a small granular assembly. However, the influence is greatly reduced for a large granular assembly, because of the averaging of the photoelastic effect over a large number of grains. More systematic experimental study employing some statistical approach is necessary to quantify the problem; that is beyond the scope of the present paper.

Light stripes, lines of brightness seen for complete particle assemblies, must come about as a result of the reinforcing effect of bright regions in individual particles which happen to be more or less coincident in location orthogonal to the light ray. The transformation of the polarised light ray passing through all the variously loaded particles is controlled by the multiplicative effects of internal stresses which bear little resemblance at the micro-scale to the macroscopic concept of stress in the granular material.

Light stripes seen in samples of crushed glass or glass spheres under modest external stresses could be the integrated effect of monochromatic fringes of less than half fringe order in some of the particles in the sample. In such a case a monotonic relationship between the stress within the strongly loaded particles and light intensity would exist. Maximum light intensity appears in Fig. 10 for about the 7th image segment (about $70 \mathrm{MPa}$ ). We cannot be sure of the level of stress generated in individual particles for any particular footing load-in principle they could be much higher than the external load and possibly exceed $70 \mathrm{MPa}$. It is probable that all the published tests made on large numbers of grains in macroscopically plane stress ( $\&$ strain) conditions known to us have been performed within this half fringe range. Image processing tools give a chance to study them in detail [25]. Classical photoelasticity is not of much use here because the local light intensity does not correspond to any overall continuum stress field.

The dimensions of the light stripes are quite different from the dimensions of small-scale strain features seen within the granular material: the strain features have a structure of the order of a few particles whereas the light stripes have a structure which appears to be of sub-particle size. In fact detailed examination of the images taken using polarised light suggests that the light stripes themselves are in some way an optical illusion which breaks down into a linear speckle when magnified. The experiments with two-dimensional assemblies (Fig. 2) and discrete element analyses (Fig. 4) indicate a typical dimension of the network of strongly loaded contacts of the order of 5 particle diameters so that one might anticipate that only one in five of particles through the thickness of the sample would be experiencing the high internal stresses. It is also evident from Fig. 10 that the bright zone within an individual particle does not extend over the full width of the particle.

The classical qualitative interpretation of light stripes in terms of major principal stress directions seems plausible. However, the quantitative interpretation of the intensity of the light transmitted in these polarised images remains uncertain.

Acknowledgments This collaboration was made possible by a British Council Research Partnership award which supported exchange visits between the two authors. The experiments conducted in Gdańsk could not have been performed without the valuable assistance of Krzysztof Gaca. The helpful comments of the reviewers are gratefully acknowledged.

Open Access This article is distributed under the terms of the Creative Commons Attribution Noncommercial License which permits any noncommercial use, distribution, and reproduction in any medium, provided the original author(s) and source are credited.

\section{Appendix: Photoelasticity: circularly polarised light}

Light from a monochromatic source passes through a linear polariser and then a quarter wave plate set with its axis at $\pi / 4$ to the axis of polarisation. We can imagine the incident light being built up of waves in horizontal $x$ direction and vertical $y$ direction, with relative phase $v$,

$E_{x}=a_{x} \cos \omega t ; \quad E_{y}=a_{y} \cos (\omega t+v)$

We can represent this as a vector, $\left(\begin{array}{ll}a_{x} & a_{y}\end{array}\right)^{T}$, so that the effect of a linear polariser with its axis at $\pi / 2$ to the horizontal is to eliminate the $x$ component of the light. This can be described by a multiplying matrix: 
$\left(\begin{array}{ll}0 & 0 \\ 0 & 1\end{array}\right)$

The effect of the quarter wave plate is to speed up one wave and to slow down the other wave by the same amount:

$\frac{1}{\sqrt{2}}\left(\begin{array}{ll}1 & i \\ i & 1\end{array}\right)$

where $i=\sqrt{-1}$. The emergent light is circularly polarised $(1 / \sqrt{2})(11)^{T}$, taking $a_{y}=1$ for convenience.

The light passing through the model under stress splits into waves vibrating in the directions of the in-plane principal stresses. (We will describe this shortly.) The light then passes through a second quarter wave plate with its axis at $\pi / 2$ to the axis of the first quarter wave plate:

$\frac{1}{\sqrt{2}}\left[\begin{array}{cc}1 & -i \\ -i & 1\end{array}\right]$

and finally through another plane polariser which can be oriented with its axis either horizontally-so that it transmits only the $x$-component of the light emerging from the quarter wave plate — or vertically — so that it transmits only the $y$-component. In the absence of any effects occurring in the model the resulting pictures would then be entirely dark and entirely bright, respectively.

For a material of thickness $h$, under plane stress with principal stresses $\sigma_{1}$ and $\sigma_{2}$, the incident light splits along the principal axes and a relative retardation $\delta$ between the two waves is introduced leading to a phase difference $\Delta=2 \pi \delta / \lambda$ where $\lambda$ is the wavelength of the light and

$\delta=h\left(n_{1}-n_{2}\right)=h C\left(\sigma_{1}-\sigma_{2}\right)$

where $C$ is the photoelastic constant, linking differences of stresses with differences of refractive index $n_{1}$ and $n_{2}$ in the two principal directions.

The transforming matrix for the light ray passing through the stressed material is:

$\mathbb{M}=\left[\begin{array}{cc}\cos \Delta / 2-i \sin \Delta / 2 \cos 2 \theta & -i \sin \Delta / 2 \sin 2 \theta \\ i \sin \Delta / 2 \sin 2 \theta & \cos \Delta / 2+i \sin \Delta / 2 \cos 2 \theta\end{array}\right]$

where $\theta$ is the angle between the direction of the major principal stress and the horizontal. The emerging light ray can be described by

$$
\left(\begin{array}{c}
\sin \Delta / 2 \exp (-i 2 \theta) \\
\cos \Delta / 2
\end{array}\right) k \exp i \omega t
$$

where $k$ is a general amplitude. The human eye responds to intensity of light, which is proportional to the square of amplitude — the frequency is high $\left(f=5.1 \times 10^{14} \mathrm{~Hz}\right)$ and the time dependent component of the wave is not of importance. The intensity $I_{q}$ that is perceived is therefore:

$I_{q}=I_{a} \sin ^{2} \Delta / 2$ where $I_{a}$ accounts for the intensity of the incident light.

If the axis of the analyser is rotated to be aligned with the vertical, one gets a background that is bright. In this case the analyser will transmit the $E_{y}$ component and the intensity $I_{q}$ is

$I_{q}=I_{a} \cos ^{2} \Delta / 2$

For both dark and bright fields the intensity is independent of $\theta$ and hence the light extinction condition $\left(I_{q}=0\right)$ is only a function of $\Delta$. Thus only isochromatics will be seen when circularly polarised light is used, with location dependent on the magnitude of the principal stress difference through (12). In the general two-dimensional case where the stress difference varies from point to point in the model and a monochromatic light source is used, looking through the analyser, black and monochromatic bands will be visible covering the whole surface of the model, the centre of each band representing a contour of constant principal stress difference. Such bands are termed fringes [14]. In the dark field arrangement the intensity is zero for $\Delta=2 m \pi$ so black (dark) fringes correspond to $0,1,2, \ldots$ wavelengths. In the bright field arrangement the intensity is zero for $\Delta=(2 m+1) \pi$ (odd multiples of halfwavelengths) with black fringes corresponding to $0.5,1.5$, 2.5 wavelengths. If we count the black fringes that appear then we can deduce the level of the principal stress difference:

$\left(\sigma_{1}-\sigma_{2}\right)=N \lambda / C h=N F_{\sigma} / h$

where $N$ is the fringe order, and $F_{\sigma}=\lambda / C$ is a material constant (see 12).

\section{References}

1. Aben, H.: Integrated photoelasticity. McGraw-Hill International Book Company, London (1979)

2. Aben, H., Guillemet, C.: Photoelasticity of glass. Springer, Berlin (1992)

3. Allersma, H.G.B.: Optical analysis of stress and strain in photoelastic particle assemblies. PhD thesis, Delft University of Technology (1987)

4. Dantu, P.: Contribution à l'étude mécanique et géométrique des milieux pulvérulents. In: Proc. 4th Int. Conf. on Soil Mechanics and Foundation Engineering, Butterworths Scientific Publications, London, 1, pp. 144-148 (1957)

5. Drescher, A.: An experimental investigation of flow rules for granular materials using optically sensitive glass particles. Géotechnique 26(4), 591-601 (1976)

6. Drescher, A., De Josselin de Jong, G.: Photoelastic verification of a mechanical model for the flow of a granular material. J. Mech. Phys. Solids 20, 337-351 (1972)

7. Duran, J.: Sands, powders, and grains: an introduction to the physics of granular materials, partially ordered systems. Springer, New York (2000)

8. Dyer, M.R.: Observation of the stress distribution in crushed glass with applications to soil reinforcement. PhD thesis, University of Oxford (1985) 
9. Geng, J., Howell, D., Longhi, E., Behringer, R.P.: Footprints in sand: the response of a granular material to local perturbation. Phys. Rev. Lett. 87(3), 035506-1-035506-4 (2001)

10. Geng, J., Reydellet, G., Clément, E., Behringer, R.P.: Green's function measurements of force transmission in 2D granular materials. Physica D 182, 274-303 (2003)

11. Haake, S.J., Patterson, E.A.: Photoelastic analysis of frozen stressed specimens using spectral-content analysis. Exp. Mech. 32(3), 266-272 (1992)

12. Hall, S.A.: A methodology for 7D warping and deformation monitoring using time-lapse seismic data. Geophysics 71, O21O31 (2006)

13. Hall, S., Muir Wood, D., Ibraim, E., Viggiani, G.: Localised deformation patterning in 2D granular materials revealed by digital image correlation. Granular Matter 12(1), 1-14 (2010)

14. Heywood, R.B.: Designing by photoelasticity. Chapman and Hall, London (1952)

15. Leśniewska, D., Muir Wood, D.: Observations of stresses and strains in a granular material. J. Eng. Mech. ASCE 135(9), 10381054 (2009)

16. Liu, C.-H., Nagel, S.R., Schecter, D.A., Coppersmith, S.N., Majumdar, S., Narayan, O., Witten, T.A.: Force fluctuations in bead packs. Science 269, 513-515 (1995)

17. MuirWood, D.: Some observations of volumetric instabilities in soils. Int. J. Solids Struct. 39(13-14), 3429-3449 (2002)

18. Ramesh, K.: Digital photoelasticity. Springer, Heidelberg (2000)

19. Russ, J.C.: The image processing handbook (4th edn). CRC Press, Boca Raton (2002)

20. Russell, A.R., Muir Wood, D.: Point load tests and strength measurements for brittle spheres. Int. J. Rock Mech. Min. Sci. 46(2), 272-280 (2009)
21. Tien, H., Paikowsky, S.: The arching mechanism on the micro level utilizing photoelastic particles. In: Fourth Int. Conference on Analysis of Discontinuous Deformation, Glasgow, Scotland, UK, pp. 317-336 (2001)

22. Tordesillas, A., Zhang, J., Behringer, R.P.: Buckling force chains in dense granular assemblies: physical and numerical experiments. Geomech. Geoeng. 4, 3-16 (2009)

23. Utter, B., Behringer, R.P.: Experimental measures of affine and nonaffine deformation in granular shear. Phys. Rev. Lett. 100, 208302-1-208302-4 (2008)

24. Viggiani, G., Hall, S.A.: Full-field measurements, a new tool for laboratory experimental geomechanics. In: Burns, S.E., Mayne, P.W. \& Santamarina, J.C. (eds.) Fourth Symposium on Deformation Characteristics of Geomaterials, IOS Press, Amsterdam, 1, pp. 3-26 (2008)

25. Voloshin, A.S., Berger, C.P.: Half-fringe photoelasticity: a new approach to whole-field stress analysis. Exp. Mech. 23(3), 304313 (1983)

26. Wakabayashi, T.: Photoelastic method for determination of stress in powdered mass. In: Proc. 7th National Conference on Applied Mechanics, Japan, pp. 153-158 (1957)

27. Wang, X.R.: Asymptotic results on the product of random probability matrices. J. Phys. A Math. Gen. 29, 3053-3061 (1996)

28. White, D.J., Take, W.A.: GeoPIV: particle image velocimetry (PIV) software for use in geotechnical testing. Manual for GeoPIV. Cambridge University Engineering Department Technical Report, D-SOILS-TR322 (2002)

29. White, D.J., Take, W.A., Bolton, M.D.: Soil deformation measurement using particle image velocimetry (PIV) and photogrammetry. Géotechnique 53(7), 619-631 (2003) 